Claremont Colleges

Scholarship@ Claremont

All HMC Faculty Publications and Research

HMC Faculty Scholarship

$1-1-1993$

\title{
Topologies and Cotopologies Generated by Sets of Functions
}

\author{
Alan Dow \\ York University \\ Melvin Henriksen \\ Harvey Mudd College \\ Ralph Kopperman \\ City College of New York \\ R. G. Woods \\ University of Manitoba
}

\section{Recommended Citation}

Dow, A., M. Henriksen, R. Kopperman, R. G. Woods. "Topologies and cotopologies generated by sets of functions." Houston Journal of Mathematics 19.4 (1993): 551-586.

This Article is brought to you for free and open access by the HMC Faculty Scholarship at Scholarship @ Claremont. It has been accepted for inclusion in All HMC Faculty Publications and Research by an authorized administrator of Scholarship @ Claremont. For more information, please contact scholarship@cuc.claremont.edu. 


\title{
TOPOLOGIES AND COTOPOLOGIES GENERATED BY SETS OF FUNCTIONS
}

\author{
A. Dow, M. Henriksen, R. Kopperman, R.G. Woods*
}

\begin{abstract}
Let $L$ be either $[0,1]$ or $\{0,1\}$ with the usual order. We study topologies on a set $X$ for which the cozero-sets of certain subfamilies $\mathcal{H}$ of $L^{X}$ form a base, and the properties imposed on such topologies by hypothesizing various order-theoretic conditions on $\mathcal{H}$. We thereby obtain useful generalizatons of extremally disconnected spaces, basically disconnected spaces, and $F$-spaces. In particular we use these tools to study the space of minimal prime ideals of certain commutative rings.
\end{abstract}

\section{Introduction.}

For over half a century topologists have studied what we now call Tychonoff topological spaces with the aid of the lattice-ordered ring $C(X)$ of continuous real-valued functions on $X$. Zero-dimensional spaces have been studied using the ring $C(X,\{0,1\})$ of continuous functions on $X$ into the Boolean ring $\{0,1\}$ (with the discrete topology). Much of the early work in this area is discussed in the influential book by Gillman and Jerison [9].

If the order-theoretic rather than the ring-theoretic properties are to be emphasized, it seems preferable to replace the lattice-ordered ring $C(X)$ by the lattice $C(X,[0,1])$ of those $f \in C(X)$ that map $X$ into the closed unit interval. Let $L$ denote either $[0,1]$ with its usual topology or $\{0,1\}$ with the discrete topology. As $L$ has a linear order, $C(X, L)$ becomes a lattice if suprema and infima are defined in the usual pointwise manner. A number of extensively studied topological properties of Tychonoff spaces may be characterized by lattice-theoretic properties of $C(X, L)$. For example, the

* The research of Dow and Woods was partially supported by grants from the Natural Science and Engineering Research Council of Canada, and that of Kopperman by PSC-CUNY grants. 
following two results are minor modifications of $3 N(6)$ in [9]. (Recall that subsets $A, B$ of $X$ are completely separated if there is an $f \in C(X,[0,1])$ such that $f[A]=\{0\}$ and $f[B]=\{1\}$.)

1.1. A Tychonoff space $X$ is extremally disconnected (i.e., each pair of disjoint open subsets of $X$ is completely separated) iff $C(X,[0,1])$ is complete as a lattice.

1.2. A Tychonoff space $X$ is basically disconnected (i.e., each pair of disjoint open sets, one of which is a cozeroset, is completely separated) iff $C(X,[0,1])$ is $\sigma$-complete iff $C(X,\{0,1\})$ is $\sigma$-complete.

The following result is essentially the same as the main result in [Se].

1.3. A Tychonoff space is an $F$-space (i.e., each pair of disjoint cozerosets is completely separated) iff whenever $\left(c_{n}\right)_{n<\omega}$ is an increasing sequence and $\left(d_{n}\right)_{n<\omega}$ is a decreasing sequence in $C(X,[0,1])$ such that $c_{n} \leq d_{m}$ whenever $n, m \in \omega$, there is an $f \in C(X,[0,1])$ such that $c_{n} \leq f \leq d_{n}$ for every $n<\omega$.

Each of the three topological properties mentioned above involves the assertion that certain pairs of disjoint subsets of a Tychonoff space $X$ are completely separated. These properties in turn imply that certain subspaces are $C^{*}$-embedded; see [9] for more details.

Each of the three classes of spaces mentioned above is characterized by imposing lattice-theoretic conditions on all of $C(X,[0,1])$ and/or $C(X,\{0,1\})$. In this paper we introduce broader classes of spaces by imposing similar lattice-theoretic conditions on subsets of these lattices, and then refining the techniques used in the proofs of 1.1-1.3 to apply in these more general conditions. Just as the classes of extremally disconnected, basically disconnected, and $F$-spaces have found many applications in algebra and functional analysis (see [9] and [16], these broader classes of spaces also arise naturally in such settings. Perhaps the most interesting application is to the study of minimal prime ideals in certain commutative rings; see Section 3 for details.

We proceed as follows. For a set $X$, let $\mathcal{H}$ denote a subset of $L^{X}$. The function with constant value $a$ is denoted by a. If $f \in \mathcal{H}$, we denote $X \backslash f^{\leftarrow}(0)$ by $\operatorname{cz}(f)$ and $f^{\leftarrow}(0)$ by $Z(f)$. (Thus $f^{\leftarrow}(1)=Z(\mathbf{1}-f)$. We impose sufficient hypotheses of $\mathcal{H}$ to ensure that $\{\operatorname{cz}(f): f \in \mathcal{H}\}$ forms a base for a topology $\tau(\mathcal{H})$ on $X$ for which $\{\operatorname{int} Z(1-h): h \in \mathcal{H}\}$ is also an open base. In general $\tau(\mathcal{H})$ is weaker than the weak topology generated on 
$X$ by $\mathcal{H}$, and not all members of $\mathcal{H}$ need be continuous with respect to it. Hence $\operatorname{cz}(f)$ need not be a cozeroset of a continuous function from $X$ to $L$ (and hence we have avoided the more common notation $\operatorname{coz}(f)$ ). Even if all members of $\mathcal{H}$ are continuous, $\{\mathrm{cz}(f): f \in \mathcal{H}\}$ may be a proper subset of the set of all cozero sets of members of $C(X)$. Observe that if $(X, \tau)$ is Tychonoff and $\mathcal{H}=C(X,[0,1])$, then $\tau(\mathcal{H})=\tau$, the original topology on $X$. As $\mathcal{H}$ inherits the order possessed by $L^{X}$, it makes sense to impose conditions on $\mathcal{H}$ similar to those imposed on $C(X,[0,1])$ in $1.1,1.2$, and 1.3 above. By doing so we cause $\tau(\mathcal{H})$ to have properties similar to, but not as strong as, being basically or extremally disconnected, or being an $F$-space.

In Section 2 we develop the theory outlined above. The section leads up to 2.13 , in which we prove a very general separation theorem. Besides unifying the proofs of familiar results (e.g., regular Lindelof spaces are normal and weakly Lindelof subspaces of $F$-spaces are $C^{*}$-embedded) usually handled by separate arguments, this result can be applied to obtain many new results. This is done in section 3 , where the machinery developed in Section 2 is used to study a class of spaces that includes both subspaces of $F$-spaces and spaces of minimal prime ideals of certain kinds of commutative rings.

Section 4 is devoted to showing that some of the spaces discussed in Section 3 cannot be embedded in a compact $F$-space. In particular, there are spaces $X$ such that the space $m X$ of minimal prime ideals of the ring $C(X)$ has no compactification that is an $F$-space. This is the case for $X=\beta \omega \backslash \omega$ if Martin's Axiom holds, and there are other such spaces in $Z F C$. This improves greatly on the results [6] where it was shown that $m X$ is not basically disconnected for these latter spaces $X$. Section 5 is devoted to miscellaneous examples and problems, and unsolved problems are posed.

\section{Topologies generated by families of functions.}

Let $L$ denote $\{0,1\}$ or $[0,1]$. If $X$ is a set, then $L^{X}$ is the complete lattice of functions from $X$ to $L$ under the usual pointwise operations $\wedge$ and $\vee$. If $f, g \in L^{X}$ and $x \in X$, then the functions $(f+g) \wedge \mathbf{1}$ and $(f-g) \vee \mathbf{0} \in$ $L^{X}$ are defined in the usual manner, i.e., $((f+g) \wedge 1)(x)=\min \{f(x)+$ $g(x), 1\}$ and $\{(f-g) \vee 0\}(x)=\max \{f(x)-g(x), 0\}$. We generalize notations involving elements to subsets of $L^{X}$; for example, $A \leq B$ means that $a \leq b$ for each $a \in A$ and $b \in B$, and $1-B$ denotes $\{1-f: f \in B\}$. (Here 0 and 1 are the obvious constant functions in $L^{X}$.) Throughout this section $\mathcal{H}$ denotes a subfamily of $L^{X}$. As usual, $f^{\leftarrow}(0)$ is denoted $Z(f)$. 
2.1. Definitions. Let $\mathcal{H} \subseteq L^{X}$, where $L=\{0,1\}$ or $[0,1]$.

(a) A subset of $X$ of the form $X \backslash f^{\leftarrow}(0)$ for some $f \in \mathcal{H}$ is called an $\mathcal{H}$-cozero-set of $X$ and is denoted $\operatorname{cz}(f)$. If $\mathbf{m}$ is an infinite cardinal, then an $\mathbf{m}-\mathcal{H}$-cozero set of $X$ is a union of fewer than $\mathbf{m} \mathcal{H}$-cozerosets of $X$.

(b) $\mathcal{H}$ is called basic if it satisfies the following conditions:

(i) $\{\mathrm{cz}(f): f \in \mathcal{H}\}$ (henceforth denoted $\mathcal{B}(\mathcal{H})$ ) is a base for a topology $\tau(\mathcal{H})$ on $X$

(ii) for each $f \in \mathcal{H}$ and $x \in \mathrm{cz}(f)$, there are $g, h \in \mathcal{H}$ such that $x \in \mathrm{cz}(g) \subseteq h^{\leftarrow}(1)$ and $\mathrm{cz}(h) \subseteq \mathrm{cz}(f)$.

(c) The topology $\tau^{*}(\mathcal{H})$ generated by $\left\{\operatorname{int}_{\tau(\mathcal{H})} Z(f): f \in \mathcal{H}\right\}$ is called the cotopology generated on $X$ by $\mathcal{H}$.

(d) A basic family $\mathcal{H}$ is called regular if $X \backslash f^{\leftarrow}(1) \in \tau(\mathcal{H})$ whenever $f \in \mathcal{H}$.

(e) A basic family $\mathcal{H}$ is completely regular if $\mathcal{H} \subseteq C(X, L)$ (where $X$ has the topology $\tau(\mathcal{H})$ ).

(f) $\mathcal{H}$ is complemented if $h \in \mathcal{H}$ implies $\mathbf{1}-h \in \mathcal{H}$.

(g) If $Y, Z \subseteq X$ and there exists $h \in \mathcal{H}$ such that $h[Y]=\{1\}$ and $h[Z]=\{0\}$, then we say that $Y$ is $\mathcal{H}$-separated from $Z$ by $h$. If one of $Y, Z$ is $\mathcal{H}$-separated from the other, we say that $Y$ and $Z$ are completely $\mathcal{H}$-separated.

If $\mathcal{H}$ is complemented, the latter two concepts coincide. Otherwise it is possible for a subset $Y$ of $X$ to be $\mathcal{H}$-separated from $Z$ without $Z$ being $\mathcal{H}$-separated from $Y$. For example, if $X=[0,1], \mathcal{H}=\operatorname{Sier}(X)$ (see $2.2(\mathrm{~d}$ ) below), $Y=(0,1)$, and $Z=\{0,1\}$, then $\chi_{Y} \in \mathcal{H}$ provides an $\mathcal{H}$-separation of $Y$ from $Z\left(\chi_{Y}\right.$ is the characteristic function of $\left.Y\right)$. But if $Z$ were $\mathcal{H}$ separated from $Y$ by some $h \in \mathcal{H}$, then $Z=h^{\leftarrow}(1)$ would be open, which is not the case.

2.2. Observations and examples. Topological operations are with respect to $\tau(\mathcal{H})$.

(a) If $\mathcal{H}$ is a basic subfamily of $L^{X}$, then $\left\{\right.$ int $\left.f^{\leftarrow}(1): f \in \mathcal{H}\right\}$ is a base for $\tau(\mathcal{H})$.

(b) If $\mathcal{H}$ is a basic subfamily of $L^{X}$, then $\tau^{*}(\mathcal{H}) \subseteq \tau(\mathcal{H})$, but in general these topologies need not be equal. As an example, let $(X, \sigma)$ be a topological space, and let $\mathcal{H}=\left\{\chi_{W}: W \in \sigma\right\}$. Then $\tau(\mathcal{H})=\sigma$, and $\mathcal{H} \subseteq C(X,\{0,1\})$ if and only if $\mathcal{H}$ is complemented if and only if each open 
set is closed (which, of course, is highly unusual). Here $\tau^{*}(\mathcal{H})$ is the topology on $X$ generated by the regular open sets of $X$, and hence $\tau(\mathcal{H})=\tau^{*}(\mathcal{H})$ if and only if $\sigma$ is a semiregular topology on $X$ (i.e. a topology for which the regular open sets form an open base).

(c) Observe that 2.1 (b) (ii) follows from 2.1 (b)(i) if $L=\{0,1\}$, as $\mathrm{cz}(f)=f^{\leftarrow}(1)$.

(d) Let $(X, \tau)$ be a topological space. Then several different basic families of functions can be defined in terms of $\tau$, as follows. Let

$\operatorname{CZe}(X)=\left\{f \in[0,1]^{X}: \operatorname{cz}(f) \in \tau\right\}$,

$\operatorname{Lsc}(X)=\left\{f \in[0,1]^{X}: f^{\leftarrow}([a, 1]) \in \tau\right.$ if $\left.0 \leq a \leq 1\right\}$,

$\operatorname{Sier}(X)=\left\{f \in\{0,1\}^{X}: \operatorname{cz}(f) \in \tau\right\}$

$C(X,\{0,1\})=\operatorname{Sier}(X) \cap(1-\operatorname{Sier}(X))$,

$C(X,[0,1])=\operatorname{Lsc}(X) \cap(1-\operatorname{Lsc}(X))$.

It is an exercise to verify that each of these families is basic (to show that $\operatorname{Lsc}(X)$ satisfies 2.1 (b) (i), note that if $f, g \in \operatorname{Lsc}(X)$, then $\chi_{\mathrm{cz}(f) \cap \mathrm{cz}(g)} \in \operatorname{Lsc}(X)$ and that $\operatorname{cz}\left(\chi_{\mathrm{cz}(f) \cap \mathrm{cz}(g)}\right)=\mathrm{cz}(f) \cap \mathrm{cz}(g)$. For the first three families, use the characteristic function $\chi_{\mathrm{cz}(f)}$ for the $g$ and $h$ required in 2.1 (b) (ii); the last two families are easily handled. Note that $C(X,\{0,1\})$ and $C(X,[0,1])$ are also complemented. Further, if $\mathcal{H}$ is any of the above five families, then $\tau(\mathcal{H}) \subseteq \tau$. On the other hand, if $V \in \tau$, it is clear that $\chi_{V}$ is in $\operatorname{Sier}(X), \operatorname{Cze}(X)$ and $\operatorname{Lsc}(X)$. Thus

$$
\tau=\tau(\operatorname{Lsc}(X))=\tau(C z e(X))=\tau(\operatorname{Sier}(X)) .
$$

In particular, every topology on a set $X$ is of the form $\tau(\mathcal{H})$ for a suitable chosen basic family $\mathcal{H}$.

Finally, observe that $\tau(C(X,\{0,1\}))$ is the topology on $X$ generated by the open-and-closed members of $\tau$, and $\tau(C(X,[0,1]))$ is the weak topology generated on $X$ by the continuous [0,1]-valued functions defined on $(X, \tau)$. Thus $(X, \tau)$ is zero-dimensional if and only if $\tau=\tau(C(X,\{0,1\}))$, and $(X, \tau)$ is completely regular if and only if $\tau=\tau(C(X,[0,1]))$.

(e) For an arbitrary space $(X, \tau)$, we define $\operatorname{Reg}(X)$ to be $\operatorname{Cze}(X) \cap$ $(1-\operatorname{Cze}(X))$. Then $\operatorname{Reg}(X)$ is complemented. However, it will not be basic in general; in fact, it is basic if and only if $\tau$ is a regular topology, and in this case $\tau(\operatorname{Reg}(X))=\tau$.

To verify this, first note that if $(X, \tau)$ is any space and if $U, V \in \tau$ with $c \ell U \subseteq V$, then the function $\frac{1}{2}\left(\chi_{c \ell U}+\chi_{V}\right)$, henceforth denoted by $f_{U, V}$, belongs to $\operatorname{Reg}(X)$; to see this, observe that $\operatorname{cz}\left(f_{U, V}\right)=V$ and $\operatorname{cz}\left(1-f_{U, V}\right)=$ 
$X \backslash c \ell U$. Also note that if $V \in \tau$, and if we define $g_{V}: X \rightarrow[0,1]$ by $g_{V}(x)=\frac{1}{2}$ if $x \in V, g_{V}(x)=0$ if $x \in X \backslash V$, then it is easy to verify that $g_{V} \in \operatorname{Reg}(X)$ and $\mathrm{cz}\left(g_{V}\right)=V$.

Now suppose $\operatorname{Reg}(X)$ is basic, and let $V \in \tau$. Then $V=\operatorname{cz}\left(g_{V}\right)$, so by 2.1 (b) (ii), if $x \in V$ there exist $g, h \in \operatorname{Reg} X$ such that $x \in \mathrm{cz}(g)$ and $h^{\leftarrow}(1)$ is $\tau$-closed. Thus $c \ell_{\tau} c z(g)$ is a closed $\tau$-neighborhood of $X$ contained in $V$. Thus $\tau$ is regular. Clearly $\tau=\tau(\operatorname{Reg}(X))$ be the above remarks.

Conversely, suppose that $\tau$ is regular. If $f, g \in \operatorname{Reg}(X)$ and $x \in$ $c z(f) \cap c z(g)$, then $V=c z(f) \cap c z(g) \in \tau$ so there exists $U \in \tau$ such that $x \in U \subseteq c l U \subseteq V$. Then $f_{U, V} \in \operatorname{Reg}(X)$ as noted above, and $x \in$ $c z\left(f_{U, V}\right)=V$. Consequently 2.2 (b) (i) is satisfied. To verify 2.1 (b) (ii), suppose $x \in c z f$ for some $f \in \operatorname{Reg}(X)$. Then $c z(f) \in \tau$ and as $\tau$ is regular, there exist $U, W \in \tau$ such that

$$
x \in W \subseteq c \ell_{\tau} W \subseteq U \subseteq c \ell_{\tau} U \subseteq c z(f) .
$$

Then $f_{U, c z(f)} \in \operatorname{Reg}(X)$ and $f_{W, U} \in \operatorname{Reg}(X)$, and one easily checks that

$$
x \in c z\left(f_{W, U}\right) \subseteq f_{U, c z(f)} \leftarrow(1) \subseteq c z\left(f_{U, c z f}\right) \subseteq c z f .
$$

Hence 2.1 (b) (ii) holds, and $\operatorname{Reg}(X)$ is regular. Again, one easily checks that $\tau(\operatorname{Reg}(X))=\tau$. Thus we have shown that $(X, \tau)$ is regular iff $\tau=\tau(\mathcal{H})$ for some symmetric (thus regular) $\mathcal{H}$.

In what follows, we will show that is $\mathcal{H}$ is a regular subfamily of $L^{X}$, then certain separated subsets of $(X, \tau(\mathcal{H}))$ can be completely $\mathcal{H}$-separated (see 2.14). When $\mathcal{H}=\{f \mid X: f \in C(Y,[0,1])\}$, and $X$ is a (dense) subspace of $Y$, we obtain previously known results concerning separation in Tychonoff spaces. Some of these results appear at the end of this section (see 2.15 and 2.16). In Section 3 we obtain new consequences of theorems 2.14 and 2.15 .

The tool that we use to show that separated subsets of $X$ can be completely separated by members of $\mathcal{H}$ will be the following. We will show that if $\mathcal{C}, \mathcal{D} \subseteq \mathcal{H}$, if $\mathcal{C} \leq \mathbf{1}-\mathcal{D}$, and if $\mathcal{C}$ and $\mathcal{D}$ are not too large, there will be an $f \in \mathcal{H}$ "in between" $\mathcal{C}$ and $\mathbf{1}-\mathcal{D}$, and this function will provide the required complete separation. We make these ideas precise in the next few definitions.

2.3. Definitions. (a) Let $\mathbf{m}, \mathbf{n}$ denote cardinal numbers. Then $\mathcal{H}$ is $(\mathbf{m}, \mathbf{n})$-nested if for each $\mathcal{C}, \mathcal{D} \subseteq \mathcal{H}$, if $|\mathcal{C}|<\mathbf{m},|\mathcal{D}|<\mathbf{n}$, and $\mathcal{C} \leq \mathbf{1}-\mathcal{D}$ 
then there is an $f \in \mathcal{H}$ such that $\mathcal{C} \leq f \leq \mathbf{1}-\mathcal{D}$. We say $\mathcal{H}$ is $(\mathbf{m}, \infty)$ nested if it is $(\mathbf{m}, \mathbf{n})$-nested for every cardinal $\mathbf{n}$.

(b) If $\mathcal{C} \subset \mathcal{D}$, a difjoin for $\mathcal{C}$ is an $f \in \mathcal{H}$ such that $\mathcal{C} \leq f$ and if $d \in \mathcal{H}$ and $\mathcal{C} \leq \mathbf{1}-d$, then $f \leq \mathbf{1}-d$.

(c) If $\mathcal{H}$ is a basic family, then $\mathrm{jo}(\mathcal{H})$ will denote the set of joins of finite subsets of $\mathcal{H}$.

It is routine to verify that if $\mathcal{H}$ is basic, then jo $(\mathcal{H})$ is basic, $(\omega, \infty)$ nested, and if $\mathcal{H}$ is $(\mathbf{m}, \mathbf{n})$-nested, then so is jo $(\mathcal{H})$. Also, if $\mathcal{H}$ is regular or completely regular, then so is $\mathrm{jo}(\mathcal{H})$.

Observe that if $\mathcal{H}$ is $(\mathbf{m}, \mathbf{n})$-nested, and if $\mathbf{p} \leq \mathbf{m}$ and $\mathbf{q} \leq \mathbf{n}$, then $\mathcal{H}$ is $(\mathbf{p}, \mathbf{q})$-nested. As the reader will observe, the concept of being $(\mathbf{m}, \mathbf{n})$-nested will be used below mostly when $\mathbf{m}=\mathbf{n}$ or $\mathbf{n}=\infty$.

2.4. Theorem. Let $\mathcal{H}$ be a basic subset of $L^{X}$ for a space $X$. The following are equivalent for a cardinal $\mathbf{m}$ :

(a) $\mathcal{H}$ is $(\mathbf{m}, \infty)$-nested.

(b) $\mathcal{H}$ is $\left(\mathbf{m},|\mathcal{H}|^{+}\right)$-nested.

(c) If $\mathcal{C} \subseteq \mathcal{H}$ and $|\mathcal{C}|<\mathbf{m}$, then $\mathcal{C}$ has a difjoin.

If in addition $\mathcal{H}$ is regular and $L=\{0,1\}$, the following is equivalent to each of the above:

(d) If $\mathcal{C} \subseteq \mathcal{H}$ and $|\mathcal{C}|<\mathbf{m}$, then the characteristic function $\chi_{A} \in \mathcal{H}$, where $A=c \ell\left[\cup\left\{f^{\leftarrow}(1): f \in \mathcal{C}\right\}\right]$. (Topological operations are with respect to $\tau(\mathcal{H}))$.

Proof. Clearly (a) implies (b). To see that (b) implies (c), if $\mathcal{C} \subseteq \mathcal{H}$ and $|\mathcal{C}|<\mathbf{m}$, let $\mathcal{D}=\{d \in \mathcal{H}: \mathcal{C} \leq \mathbf{1}-d\}$. Then $\mathcal{C}, \mathcal{D} \subseteq \mathcal{H}, \mathcal{C} \leq \mathbf{1}-\mathcal{D},|\mathcal{C}|<\mathbf{m}$, and $|\mathcal{D}|<|\mathcal{H}|^{+}$, so for some $f \in \mathcal{H}$ we have $\mathcal{C} \leq f \leq \mathbf{1}-\mathcal{D}$. This $f$ is a difjoin for $\mathcal{C}$, since if $d \in \mathcal{H}, \mathcal{C} \leq 1-d$, then $d \in \mathcal{D}$, so $\mathcal{C} \leq f \leq 1-d$. To see that (c) implies (a), suppose each subset $\mathcal{C}$ of $\mathcal{H}$ of cardinality less than $\mathbf{m}$ has a difjoin $f$. For such $\mathcal{C}, f$, if $\mathcal{D} \subseteq \mathcal{H}$ and $\mathcal{C} \leq \mathbf{1}-\mathcal{D}$ then for each $d \in \mathcal{D}, \mathcal{C} \leq \mathbf{1}-d$; thus $\mathcal{C} \leq f \leq \mathbf{1}-\mathcal{D}$ and so $\mathcal{H}$ is $(m, \infty)$-nested.

To show (a) implies (d), note that the regularity of $\mathcal{H}$ implies that if $h \in \mathcal{H}$ then $h^{\leftarrow}(0)$ and $h^{\leftarrow}(1)$ are both $\tau(\mathcal{H})$-closed; hence $\mathcal{H} \subseteq C(X,\{0,1\})$, and if $d \in \mathcal{H}$ then $\mathbf{1}-d \in C(X,\{0,1\})$.

Suppose (a) holds. For each $x \in X \backslash A$ there exists $r_{X} \in \mathcal{H}$ such that $x \in r_{X}^{\leftarrow}(1) \subseteq X \backslash A$. Thus $f^{\leftarrow}(1) \cap r_{X}^{\leftarrow}(1)=\phi$ for each $f \in \mathcal{H}$ and $x \in X \backslash A$. 
Since $f$ and $r_{X}$ are $\{0,1\}$-valued, it follows that $\mathcal{C} \leq 1-\left\{r_{X}: x \in X \backslash A\right\}$. By (a) there exists $h \in \mathcal{H}$ such that $f \leq h \leq 1-r_{X}$ for each $f \in \mathcal{H}$ and $x \in X \backslash A$. Thus $h\left[\cup\left\{f^{\leftarrow}(1): f \in \mathcal{C}\right\}\right]=\{1\}$, so by continuity of $h, h[A]=$ $\{1\}$. If $x \in X \backslash A$, then $h(x) \leq\left(1-r_{X}\right)(x)=0$; hence $h[X \backslash A]=\{0\}$. Thus $h=\chi_{A}$.

Now suppose (d) holds, and let $\mathcal{C} \subseteq \mathcal{H}$ such that $|\mathcal{C}|<\mathbf{m}$. We will show that $\mathcal{C}$ has a difjoin, and hence (c) holds. Suppose $\mathcal{D} \subseteq \mathcal{H}$ and $\mathcal{C} \leq \mathbf{1}-\mathcal{D}$. By hypothesis $\chi_{A} \in \mathcal{H}$. Observe that if $f \in \mathcal{C}$, and if $f(x)=1$, then $x \in A$ so $\chi_{A}(x)=1$. As $f$ and $\chi_{A}(x)$ are $\{0,1\}$-valued, it follows that $\mathcal{C} \leq f$.

Now suppose that $d \in \mathcal{D}$ and $x \in X$. If $d(x)=0$, then $(1-d)(x)=1$ and so $\chi_{A}(x) \leq(1-d)(x)$. If $d(x)=1$, then $x \notin A$; for if $x \in A$ then as $d^{\leftarrow}(1)$ is open (since $\mathcal{H} \subset C(X,\{0,1\})$, there would exist $f \in \mathcal{C}$ such that $f^{\leftarrow}(1) \cap d^{\leftarrow}(1) \neq \phi$. If $y \in f^{\leftarrow}(1) \cap d^{\leftarrow}(1)$, then $1=f(y) \leq(1-d)(y)=0$, a contradiction. Thus $x \notin A$, so $\chi_{A}(s)=0 \leq(1-d)(x)$. Thus $\chi_{A} \leq 1-\mathcal{D}$, and hence $\chi_{A}$ is a difjoin of $\mathcal{C}$, and so (c) holds.

2.5. Comments and examples. We have several examples.

(a) Difjoins need not be unique and need not be joins. For example, suppose that $\mathcal{H}$ is the set of non-decreasing functions from $[0,1]$ onto $[0,1]$. Then $\tau(\mathcal{H})=\{(a, 1]: a \in[0,1]\} \cup\{[a, 1]: a \in[0,1]\}$, and it follows that $\mathcal{H}$ is a basic subset of $[0,1]^{[0,1]}$. Observe that $\tau^{*}(\mathcal{H})$ is the indiscrete topology. If $c, d \in \mathcal{H}$ it is not possible for $c \leq 1-d$ to hold. Hence if $\mathcal{C} \subseteq \mathcal{H}$ and $f$ is a join for $\mathcal{C}$, then (vacuously) $\mathbf{r} f \wedge \mathbf{1}$ is a difjoin for $\mathcal{C}$ for each $\mathbf{r} \geq \mathbf{1}$. As another example, let $\mathcal{H}$ be the constant function 1 together with the set of characteristic functions on finite subsets of $[0,1]$. Then $\mathcal{H}$ is a basic subset of $[0,1]^{[0,1]}, \mathbf{1}$ is the join of any countably infinite subset of $\mathcal{H} \backslash\{\mathbf{1}\}$, but such a set has no difjoin in $\mathcal{H}$. On the other hand, if $\mathcal{H}$ is complemented then difjoins and joins are identical.

More generally, if $\mathcal{C} \subseteq \mathcal{H}$ has a difjoin $g$, and a join $\vee \mathcal{C}$, then $\vee \mathcal{C}$ is a difjoin for $\mathcal{C}$ (since if $h \in \mathcal{H}$, then $\mathcal{C} \leq \vee \mathcal{C} \leq g \leq 1-h$ ). If further $\mathcal{H} \cup(\mathbf{1}-\mathcal{H}) \subseteq \mathcal{H} \subseteq L^{X}$, then each $\mathcal{H}$-join in $\mathcal{H}$ is a $\mathcal{H}$-difjoin (and an $\mathcal{H}$-join) (since if $\mathcal{D} \leq \mathbf{1}-h, \mathcal{D} \leq \vee \mathcal{D} \leq \mathbf{1}-h$, where $\vee \mathcal{D}$ denotes $\mathcal{H}$-join of $\mathcal{D})$.

(b) Recall (see for example [2]) that a zero-dimensional topological space $X$ is $\mathbf{m}$-basically disconnected if, whenever $\mathcal{F}$ is a family of fewer than $\mathbf{m}$ clopen subsets of $X$, then $\operatorname{cl}_{X}(\cup \mathcal{F})$ is clopen in $X$. (A different definition is given in [26] for arbitrary (Tychonoff) spaces which coincides 
with this one for strongly zero-dimensional spaces). If $\mathbf{m}=\omega$ the condition holds automatically, and if $\mathbf{m}=\omega_{1}, X$ is called a basically disconnected space (see [23] or [9]). It is clear from the equivalence of (a) and (d) in 2.4 above that $X$ is $\mathbf{~ m}$-basically disconnected if and only if $C(X,\{0,1\})$ is $(\mathbf{m}, \infty)$-nested.

More generally, suppose that $X$ is zero-dimensional Hausdorff space and has an $\mathbf{m}$-basically disconnected compactification $\alpha X$. Let $\mathcal{H}=\{f \mid$ $X: f \in C(\alpha X,\{0,1\})\}$; then $\mathcal{H}$ is a complemented regular subset of $\{0,1\}^{X}$ and clearly $\mathcal{H}$ is $(\mathbf{m}, \infty)$-nested. Conversely, suppose $\mathcal{H}$ is a complemented regular $(\mathbf{m}, \infty)$-nested subset of $\{0,1\}^{X}$. Then $\left\{h^{\leftarrow}(1): h \in \mathcal{H}\right\}$ is a Boolean algebra $\mathcal{B}$ of clopen sets of $X$ that forms a base for the closed sets of $X$. By 2.4, the fact that $\mathcal{H}$ is $(\mathbf{m}, \infty)$-nested means that $\mathcal{B}$ is an $\mathbf{m}$-complete Boolean algebra, as $\vee\left\{h^{\leftarrow}(1): h \in \mathcal{F}\right\}=c l\left[\cup\left\{h^{\leftarrow}(1): h \in \mathcal{F}\right\}\right]$ if $\mathcal{F} \subseteq \mathcal{B}$ and $|\mathcal{F}|<\mathbf{m}$. The Stone space of $\mathcal{B}$ is a compactification of $X$ (see, for example, $4 \mathrm{I}$ of [23]), and it will be $\mathbf{m}$-basically disconnected since $\mathcal{B}$ is $\mathbf{m}$-complete. For background on this latter, see [2] and [26].

In summary, we have shown that a zero-dimensional Hausdorff space $X$ has a complemented regular subfamily $\mathcal{H}$ of $\{0,1\}^{L}$ that is $(\mathbf{m}, \infty)$-nested if and only if $X$ has an $\mathbf{m}$-basically disconnected compactification. (It is well-known that not all dense subspaces of basically disconnected spaces need be basically disconnected; see example 1 of [18].)

(c) As a special case of (b), recall that a Hausdorff space $X$ is $e x$ tremally disconnected if its open sets have open closures. It is clear from the preceding discussion that a Hausdorff space $X$ is extremally disconnected if and only if $C(X,\{0,1\})$ is $(\mathbf{m}, \infty)$-nested for every $\mathbf{m}$.

(d) Recall from 1.3 above that a Tychonoff space $X$ is an $F$-space if and only if whenever $\left(c_{n}\right)$ is an ascending sequence of members of $C^{*}(X),\left(d_{n}\right)$ is a descending sequence of members of $C^{*}(X)$, and $c_{n} \leq d_{n}$ for each $n \in \omega$, there exists an $f \in C^{*}(X)$ such that $c_{n} \leq f \leq d_{n}$ for each $n \in \omega$. From this it follows quickly that $X$ is an $F$-space if and only if $C(X,[0,1])$ is $\left(\omega^{+}, \omega^{+}\right)$-nested.

More generally, suppose that $X$ is a subspace of an $F$-space $Y$. Then $\beta Y$ is an $F$-space (14.25 of [9]) and so its $C^{*}$-embedded subspace $\operatorname{cl}_{\beta Y} X$ is an $F$-space that contains $X$ as a dense subspace (see 14.26 of [9]). Let $\mathcal{H}=$ $\left\{f \mid X: f \in C^{*}\left(\operatorname{cl}_{\beta Y} X\right)\right\}$; then $\mathcal{H}$ is a regular, complemented, $\left(\omega^{+}, \omega^{+}\right)$nested subfamily of $C^{*}(X)$; in fact, it is a regular subring of $C^{*}(X)$ in the 
terminology introduced below.

Conversely, suppose that $X$ is a Tychonoff space and that $\mathcal{A}$ is a subring of $C^{*}(X)$ (the family of bounded real-valued continuous functions on $X)$. We call $\mathcal{A}$ regular if it is a complete (with respect to the sup norm metric on $C^{*}(X)$ ) subring $C^{*}(X)$ containing the constant $\mathbb{R}$-valued functions, and such that $\{Z(f): f \in \mathcal{A}\}$ is a closed base for $X$. In $4.5(\mathrm{~m})$ of [23] it is shown that the maximal ideal space of $m_{\mathcal{A}} X$ of $X$ is a compactification of $X$, each $f \in \mathcal{A}$ extends continuously to $f^{\mathcal{A}} \in C^{*}\left(m_{\mathcal{A}} X\right)$, and $f \rightarrow f^{\mathcal{A}}$ is a ring isomorphism $\lambda$ from $\mathcal{A}$ onto $C^{*}\left(m_{\mathcal{A}} X\right)$. As $\lambda$ is necessarily an order isomorphism, it is clear that if $\mathcal{A}$ is $\left(\omega^{+}, \omega^{+}\right)$-nested, then $C^{*}\left(m_{\mathcal{A}} X\right)$ will be also; hence as noted above, $m_{\mathcal{A}} X$ will be an $F$-space.

In summary, a Tychonoff space $X$ is a subspace of an $F$-space iff $C^{*}(X)$ has a regular subring that is $\left(\omega_{1}, \omega_{1}\right)$-nested. Not all subspaces of $F$-spaces are $F$-spaces; in Example 3 of [18] an extremally disconnected space with a closed subspace that is not an $F$-space is given. (This corrects an inessential error in Section 2 of [Se].) Also observe that in constrast to Tychonoff $\mathbf{m}$-basically disconnected spaces, $F$-spaces need not be zerodimensional.

In what follows it will be useful to have a simple criterion for establishing the function-theoretic hypothesis involved in the definition of difjoins.

2.6. Proposition. Let $X$ be a space and let $\mathcal{H} \subseteq L^{X}$, where $L=\{0,1\}$ or $[0,1]$. Let $\mathcal{F}, \mathcal{G} \subset \mathcal{H}$.

(a) If $c z(f) \cap c z(g)=\phi$ whenever $f \in \mathcal{F}$ and $g \in \mathcal{G}$, then $\mathcal{F} \leq \mathbf{1}-\mathcal{G}$.

(b) If $L=\{0,1\}$, then the converse of (a) holds.

Proof. (a) Let $x \in X$. If $f(x)=0$, then $f(x) \leq(1-g)(x)$. If $f(x)>0$, then $x \in \mathrm{cz}(f) \subseteq X \backslash \mathrm{cz}(g)$, so $f(x) \leq 1=(1-g)(x)$.

(b) Let $f \in \mathcal{F}, g \in \mathcal{G}$, and suppose $f \leq \mathbf{1}-g$. If $x \in \mathrm{cz}(f)$, then $f(x)=1$, so $(1-g)(x)=0$. Hence $g(x)=0$ and $x \notin \mathrm{cz}(g)$.

In Section 3, we will consider spaces with a regular subfamily $\mathcal{H}$ of $L^{X}$ that is not complemented, but which is $(\mathbf{m}, \infty)$-nested. It will be shown in Section 4 that the lack of complementation means that these spaces need not have $F$-space compactifications, but the $(\mathbf{m}, \infty)$-nesting results in the spaces having many properties that one normally associates with $F$-spaces or basically disconnected spaces. 
The notions of $[\mathbf{p}, \mathbf{q}]$-compactness and final $\mathbf{p}$-compactness introduced in 2.7 below are discussed at length in [25], but from a perspective quite different from the one we shall take. Our terminology is borrowed from (and generalized from) that in [25].

Henceforth we denote the discrete topology by $\delta$.

Recall that a space $X$ is weakly Lindelöf if each open cover of $X$ has a countable subfamily whose union is dense. As noted above, weakly Lindelöf subspaces of $F$-spaces are $C^{*}$-embedded. We introduce a natural generalization of weakly Lindelöf as follows:

2.7. Definition. Let $(X, \tau)$ denote a topological space, $\mathbf{p}, \mathbf{q}$ infinite cardinals such that $\mathbf{p} \leq \mathbf{q}$, and let $\tau^{\prime}$ denote a topology on $X$ for which $\tau \subseteq \tau^{\prime}$. Then:

(a) $(X, \tau)$ is called ( $\left.\mathbf{p}, \mathbf{q}, \tau^{\prime}\right)$-compact if every $\tau$-open cover of cardinality no greater than $\mathbf{q}$ has a subfamily of cardinality less than $\mathbf{p}$ whose union is $\tau^{\prime}$-dense in $X$. It is called $\left(\mathbf{p}, \infty, \tau^{\prime}\right)$-compact if it is $\left(\mathbf{p}, \mathbf{q}, \tau^{\prime}\right)$-compact for all $\mathbf{q} \geq \mathbf{p}$.

(b) $(X, \tau)$ is called $(\mathbf{p}, \mathbf{q})$-compact if it is (p, $\mathbf{q}, \delta)$-compact, and is called finally $\mathbf{p}$-compact if it is $(\mathbf{p}, \mathbf{q})$-compact for all infinite cardinals $\mathbf{q}$ (equivalently, if it is $(p,|\mathbf{X}|)$-compact).

(c) $(X, \tau)$ is called $(\mathbf{p}, \mathbf{q})$-weakly Lindelöf if it is $(\mathbf{p}, \mathbf{q}, \tau)$-compact, and is $\mathbf{p}$-weakly Lindelöf if it is $(\mathbf{p}, \mathbf{q})$-weakly Lindelöf for all cardinals $\mathbf{q}$.

The topology $\tau^{\prime}$ will be used below only when $\tau^{\prime}=\tau$ or $\tau^{\prime}=\delta$. It enables us to handle simultaneously subfamilies of a cover of a space whose union is dense or whose union is the whole space.

Many of the above concepts have more familiar names in the countable case, as is illustrated in the following table, where $\tau$ denotes a topology on a space $X$. The reader should refer to this table to get a quick translation of unfamiliar terminology. 


$$
\begin{aligned}
& (\omega, \omega) \text {-compact } \quad=(\omega, \omega, \delta) \text {-compact } \quad=\text { countably compact } \\
& \text { finally } \omega \text {-compact } \quad=(\omega, \omega, \delta) \text { compact }=\text { compact } \\
& (\omega, \omega) \text {-weakly Lindelöf }=(\omega, \omega, \tau) \text {-compact }=\text { feebly compact } \\
& \text { (三 pseudocompact for } \\
& \text { Tychonoff spaces) } \\
& \omega \text {-weakly Lindelöf } \quad=(\omega, \infty, \tau) \text {-compact }=H \text {-closed } \\
& \text { finally } \omega^{+} \text {-compact } \quad=\left(\omega^{+}, \infty, \delta\right) \text {-compact }=\text { Lindelöf } \\
& \omega^{+} \text {-weakly Lindelöf } \quad=\left(\omega^{+}, \infty, \tau\right) \text {-compact }=\text { weakly Lindelöf. }
\end{aligned}
$$

See 1.1 and 4.8 of [23]

2.8. Proposition. Let $X$ be a space and $\mathcal{H}$ be an $(\mathbf{m}, \mathbf{n})$-nested subset of $L^{X}$, where $\mathbf{m}$ and $\mathbf{n}$ are infinite cardinals. Let $\tau=\tau(\mathcal{H})$, and let $Y$ and $Z$ be subsets of $X$ contained in disjoint members of $\tau$. Suppose further that $Z$ is $\mathbf{n}$-compact. Then there exists $h \in \mathcal{H}$ for which $Y \subseteq Z(\mathbf{1}-h)$ and $Z \subseteq Z(h)$, provided that either:

(i) $Y$ is finally $\mathbf{m}$-compact, or

(ii) $\mathcal{H}$ is regular and $Y$ is $\mathbf{m}$-weakly Lindelof.

Proof. Let $P$ and $Q$ be disjoint members of $\tau$ containing $Y$ and $Z$ respectively. Let $\tau^{\prime}=\tau$ (for part (i)), or $\tau^{\prime}=\delta$ (for part (ii)).

As $\mathcal{H}$ is basic, we can find $\mathcal{P} \subseteq \mathcal{H}$ such that $Y \subseteq \cup\left\{\right.$ int $_{X} f^{\leftarrow}(1): f \in$ $\mathcal{P}\} \subseteq \cup\{\operatorname{cz}(f): f \in \mathcal{P}\} \subseteq P$. Since $Y$ is $\left(\mathbf{m}, \infty, \tau^{\prime}\right)$ compact, there exists $\mathcal{F} \subseteq \mathcal{P}$ such that $|\mathcal{F}|<\mathbf{m}$ and

$$
Y^{\prime}=Y \cap\left[\cup\left\{\operatorname{int}_{X} f^{\leftarrow}(1): f \in \mathcal{F}\right\}\right]
$$

is $\tau^{\prime}$-dense in $Y$. Similarly we can find $\mathcal{G} \subseteq \mathcal{H}$ such that $|\mathcal{G}|<\mathbf{n}$ and

$$
Z^{\prime}=Z \cap\left[\cup\left\{\operatorname{int}_{X} g^{\leftarrow}(1): g \in \mathcal{G}\right]\right.
$$

is $\tau$-dense in $Z$, while $\cup\{c z(g): g \in \mathcal{G}\} \subseteq Q$. As $P \cap Q=\phi$, it follows that $\operatorname{cz}(f) \cap \operatorname{cz}(g)=\phi$ for each $f \in \mathcal{F}$ and $g \in \mathcal{G}$, and so by $2.6 \mathcal{F} \leq 1-\mathcal{G}$. As $\mathcal{H}$ 
is (m, n)-nested there exists $h \in \mathcal{H}$ such that $\mathcal{F} \leq h \leq \mathbf{1}-\mathcal{G}$. If $x \in Y^{\prime}$, then $1=f(x) \leq h(x)$ for each $f \in \mathcal{F}$, so $h\left[Y^{\prime}\right]=\{1\}$. If $x \in Z^{\prime}$, then $g(x)=1$ so $h(x) \leq(1-g)(x)=0$ for each $g \in \mathcal{G}$. Hence $h\left[Z^{\prime}\right]=\{0\}$, and as $h^{-}(0)$ is closed and $Z^{\prime}$ is dense in $Z, h[Z]=0$. Thus $h$ separates $Y^{\prime}$ and $Z$.

In case (i), $Y^{\prime}=Y$ and we are done. In case (ii), $Y^{\prime}$ is $\tau$-dense $Y, Y^{\prime} \subseteq Z(\mathbf{1}-h)$ which is closed since $\mathcal{H}$ is assumed to be regular, and so $Y \subseteq Z(1-h)$.

In 2.8 we gave a condition under which sets contained in disjoint open sets can be completely $\mathcal{H}$-separated. In 2.14 below we give sufficient conditions for separated sets to be completely $\mathcal{H}$-separated.

2.9. Lemma. Let $X$ be a space and let $\mathcal{H}$ be a basic subfamily of $L^{X}$. Suppose that $\mathcal{C} \subset \mathcal{H}$ and let $f$ be a difjoin for $\mathcal{C}$. Then

$$
\begin{array}{ll} 
& \text { int } Z(f)=\operatorname{int}[\cap\{Z(c): c \in \mathcal{C}\}] \\
\text { and } & \text { int } f^{\leftarrow}(1) \supseteq \cup\left\{\operatorname{int} c^{\leftarrow}(1): c \in \mathcal{C}\right\} .
\end{array}
$$

Proof. Let $S=\operatorname{int}[\cap\{Z(c): c \in \mathcal{C}\}]$. If $x \in S$, there exists $r_{x} \in \mathcal{H}$ such that $x \in$ int $r_{x}^{\leftarrow}(1) \subseteq \operatorname{cz}\left(r_{x}\right) \subseteq S$ (since $\mathcal{H}$ is basic). Suppose $y \in X$ and $r_{x}(y)>0$. Then $y \in S$ so $c(y)=0$ for each $c \in \mathcal{C}$. Thus $c(y) \leq\left(1-r_{x}\right)(y)$. If $r_{x}(y)=0$ then $c(y) \leq 1=\left(1-r_{x}\right)(y)$. Thus $\mathcal{C} \leq \mathbf{1}-\mathcal{D}$, where $\mathcal{D}=$ $\left\{r_{x}: x \in S\right\}$. As $f$ is a difjoin for $\mathcal{C}$, it follows that $c \leq f \leq \mathbf{1}-r_{x}$ for each $c \in \mathcal{C}$ and $x \in S$. Thus if $x \in S$, then $f(x) \leq\left(1-r_{x}\right)(x)=0$ so $x \in Z(f)$. Hence $S \subseteq Z(f)$, so $S \subseteq$ int $Z(f)$. Conversely, if $y \in Z(f)$ and $c \in \mathcal{C}$, then $0 \leq c(y) \leq f(y)=0$, so $y \in \cap\{Z(c): c \in \mathcal{C}\}$. Consequently int $Z(f) \subseteq S$. Finally, if $c \in \mathcal{C}$ and $x \in c^{\leftarrow}(1)$, then $1=c(x) \leq f(x) \leq 1$, so $x \in f^{\leftarrow}(1)$. Hence int $f^{\leftarrow}(1) \supseteq \cup\left\{\right.$ int $\left.c^{\leftarrow}(1): c \in \mathcal{C}\right\}$.

2.10. Lemma. Let $X$ be a space and let $\mathcal{C}, \mathcal{D} \subseteq L^{X}$. Suppose that $s, t \in L^{X}$ and that either $\mathcal{C} \leq s$ or $\mathcal{D} \leq t$. Then, for each $c \in \mathcal{C}, d \in \mathcal{D}$, we have

$$
Z((\mathbf{1}-(c-t)) \vee \mathbf{0}) \cap Z((\mathbf{1}-(d-s)) \vee 0)=\phi .
$$

Proof. It is routine to verify that $Z((\mathbf{1}-(h-k)) \vee \mathbf{0})=Z(\mathbf{1}-h) \cup Z(k)$ whenever $h, k \in L^{X}$. From this it follows that

$$
Z((\mathbf{1}-(c-t)) \vee \mathbf{0}) \cap Z((\mathbf{1}-(d-s)) \vee \mathbf{0})=Z(\mathbf{1}-c) \cap Z(t) \cap Z(\mathbf{1}-d) \cap Z(s) \text {. }
$$

Since $Z(\mathbf{1}-c) \cap Z(s)=\phi$ if $\mathcal{C} \leq s$ and $Z(\mathbf{1}-d) \cap Z(t)=\phi$ if $\mathcal{D} \leq t$, the lemma follows. 
2.11. Definition. Suppose $(X, \tau)$ is a topological space, $\mathbf{p}$ an infinite cardinal and $\tau^{\prime}$ a topology on $X$ such that $\tau \subseteq \tau^{\prime}$.

(a) $(X, \tau)$ is $\left(\mathbf{p}, \tau^{\prime}\right)$-additive, if whenever $S$ is a collection of fewer that $p$ subsets of $X$, then $\operatorname{int}_{\tau}(\cap S)$ is $\tau^{\prime}$-dense in $\cap\left\{\operatorname{int}_{\tau}(s): s \in S\right\}$.

(b) $X$ is $\mathbf{p}$-additive if $X$ is $(\mathbf{p}, \delta)$-additive.

(c) $X$ is almost $\mathbf{p}$-additive if $X$ is $(\mathbf{p}, \tau)$-additive.

All topologies are $\omega$-additive, and if $\tau$ is $\omega^{+}$-additive, then $(X, \tau)$ is called a $P$-space (see [9] or [23]). If $\tau$ is $\mathbf{m}$-additive, then $(X, \tau)$ is often called a $P_{m}$-space. Clearly if $(X, \tau)$ is any space, $\mathbf{m}$ is an infinite cardinal, and $\tau_{\mathbf{m}}=\left\{V \subseteq X\right.$ : there exists $\mathbf{k}<\mathbf{m}$ and $\left\{W_{i}: i<\mathbf{k}\right\} \subset$ $\tau$ such that $V=\cap\left\{W_{i}: i<\mathbf{k}\right\}$, then $\tau_{\mathbf{m}}$ is an $\mathbf{m}$-additive topology on $X$. Clearly if the pseudocharacter of $(X, \tau)$ is less than $\mathbf{m}$, then $\tau_{\mathbf{m}}$ is the discrete topology (see [17] or [23] for a discussion of pseudocharacter). An important class of $\mathbf{m}$-additive topologies without isolated points is the class of spaces of the form $\left(\{0,1\}^{\mathbf{m}}, \tau_{\mathbf{m}}\right)$, where $\tau$ is the usual product topology on the generalized Cantor set $\{0,1\}^{\mathbf{m}}$; see chapter 15 of [2] for details.

Let $X$ be Tychonoff. Then $X$ is almost $\omega^{+}$-additive if $X$ is an almost $P$-space (see [20]).

Finally, it is evident from the equivalence of $2.4(\mathrm{a})$ and $2.4(\mathrm{~d})$ that if $\tau$ is $\mathbf{m}$-additive, then $C(X,\{0,1\})$ is $(\mathbf{m}, \infty)$-nested.

2.12. Lemma. (a) Let $\tau^{\prime}=\tau$ or $\delta$. Suppose $(X, \tau)$ is a $\left(\mathbf{p}, \mathbf{q}, \tau^{\prime}\right)$-compact space. If $A$ is a closed subspace of $X$ and int $\tau^{\prime} A$ is $r^{\prime}$-dense in $A$ (i.e., $A$ is $\tau^{\prime}$-regular closed), then $A$ is ( $\left.\mathbf{p}, \mathbf{q}, \tau^{\prime}\right)$-compact.

(b) If $\mathbf{p}$ is regular, then a union of fewer than $\mathbf{p}\left(\mathbf{p}, \mathbf{q}, \tau^{\prime}\right)$-compact subsets of a space $(X, \tau)$ is $\left(\mathbf{p}, \mathbf{q}, \tau^{\prime}\right)$-compact.

Proof. (a) Suppose $\mathcal{C}$ is an open cover of $A$ with at most q elements. Then $\mathcal{C}^{\prime}=\mathcal{C} \cup\{(X \backslash A)\}$ is an open cover of $X$ also of cardinality no greater than $\mathbf{q}$, so there is a subfamily $\mathcal{D}^{\prime}$ of cardinality less than $\mathbf{p}$ whose union is $\tau^{\prime}$-dense in $X$. Thus if $\mathcal{D}=\mathcal{D}^{\prime} \backslash\{(X \backslash A)\}$, then $\mathcal{D}$ has cardinality less than $p$, and it suffices to show that $\cup \mathcal{D}$ is $\tau^{\prime}$-dense in $A$. But

$$
\begin{aligned}
\operatorname{int}_{\tau^{\prime}} A \subseteq \operatorname{cl}_{\tau^{\prime}}(\cup \mathcal{D} \cup(X \backslash A)) & =\operatorname{cl}_{\tau^{\prime}}(\cup \mathcal{D}) \cup \operatorname{cl}_{\tau^{\prime}}(X \backslash A) \\
& =\operatorname{cl}_{\tau^{\prime}}(\cup \mathcal{D}) \cup\left(X \backslash \operatorname{int}_{\tau^{\prime}} A\right),
\end{aligned}
$$

so int ${ }_{\tau^{\prime}} A \subseteq \mathrm{cl}_{\tau^{\prime}}(\cup \mathcal{D})$, whence $A \subseteq \mathrm{cl}_{\tau^{\prime}} \operatorname{int}_{\tau^{\prime}} A \subseteq \operatorname{cl}_{\tau^{\prime}}(\cup \mathcal{D})$. 
(b) Suppose $\mathbf{r}<\mathbf{p}$. If, for $k<\mathbf{r}, Y_{k}$ is $\left(\mathbf{p}, \mathbf{q}, \tau^{\prime}\right)$-compact, and $\mathcal{C}$ is an open cover of $Y=U\left\{Y_{k}: k<\mathbf{r}\right\}$ with at most q elements, then for each $k$ there is a subset $\mathcal{D}_{k}$ of $\mathcal{C}$ containing fewer than $\mathbf{p}$ elements and whose union is $\tau^{\prime}$-dense in $Y_{k}$. By the regularity of the cardinal $\mathbf{p}, \mathcal{D}=\cup\left\{\mathcal{D}_{k}: k<\mathbf{r}\right\}$ has fewer than $\mathbf{p}$ elements, and for each $k, Y_{k} \subseteq \operatorname{cl}_{\tau^{\prime}}\left(\cup \mathcal{D}_{k}\right) \subseteq \operatorname{cl}_{\tau^{\prime}}(\cup \mathcal{D})$.

The following lemma (2.13) is technical in nature and is motivated by theorem 2.14, which will be used in section 3. Because of this we state and prove 2.14 before giving the proof of 2.13. Note that 2.14(a) is well-known.

2.13. Theorem. Suppose $\mathcal{H}$ is an $(\mathbf{m}, \infty)$-nested basic regular subset of $L^{X}$. Let $\sigma$ and $\rho$ be topologies such that $\tau \subseteq \sigma$ and $\tau \subseteq \rho$ (where $\tau=\tau(\mathcal{H})$ ), and suppose $\tau$ is $(\mathbf{m}, \rho)$-additive. Suppose further that $Y_{1}, Y_{2}$ are $\left(\mathbf{m}^{+}, \infty, \sigma\right)$-compact $\tau$-separated subsets of $X$ (see 2.7 (a) for notation). Then there are $\sigma$-dense $\left(\mathrm{m}^{+}, \infty, \sigma\right)$-compact subsets of the $Y_{i}$ contained in the $\rho$-closures of disjoint $\tau$-open sets.

2.14. Theorem. (a) Regular Lindelöf spaces are normal.

(b) If $\mathcal{H}$ is completely regular $(\omega, \infty)$-nested, then separated weakly Lindelöf subspaces of $X$ contain dense weakly Lindelöf subspaces which are contained in disjoint open sets.

(c) If $\mathcal{H}$ is completely regular and $\left(\omega^{+}, \omega^{+}\right)$-nested, then separated weakly Lindelöf subspaces of $X$ are completely separated.

Proof of 2.14. (a) Let $Y_{1}, Y_{2}$ denote disjoint closed subspaces of the Lindelöf (i.e., $\left(\omega^{+}, \infty, \delta\right)$-compact) space $X$. As is well-known, each of these closed subspaces is Lindelöf. Notice that since $\operatorname{Reg}(X)$ is closed under finite joins, it is $(\omega, \infty)$-nested. Let $\mathcal{H}=\operatorname{Reg}(X), \mathbf{m}=\omega, \sigma=\rho=\delta$; since all spaces are $\omega$-additive, the conditions 2.13 hold. Hence ( $\delta$-dense subsets of) the $Y_{i}$ 's are contained in (the $\sigma$-closures of) disjoint open sets.

(b) Here we apply 2.13 in the case $\mathbf{m}=\omega, \sigma=\tau, \rho=\delta$, and recall that weakly Lindelöf spaces are $\left(\omega^{+}, \infty, \tau\right)$-compact.

(c) This follows by applying 2.13 to jo $(\mathcal{H})$ (see $2.3 \mathrm{ff}$.) when $\mathbf{m}=$ $\omega, \sigma=\tau, \rho=\delta$, thus obtaining disjoint open $P_{i}$ containing ( $\delta$-closures of) dense weakly Lindelöf subsets of $Y_{i}$. Then apply $2.8(\mathrm{~b})$ and recall that since $\mathcal{H}$ is completely regular, completely $\mathcal{H}$-separated subsets are completely separated.

Proof of 2.13. As $\mathcal{H}$ is basic and regular, there are for $i=1,2$, cardinals $\mathbf{p}_{i}$ and subfamilies $\mathcal{G}_{i}=\left\{\left(f_{i j}, g_{i j}\right): j<\mathbf{p}_{i}\right\}$ of $\mathcal{H} \times \mathcal{H}$ such that $Y_{i} \subseteq$ 
$\cup\left\{\operatorname{cz}\left(f_{i j}\right): j<\mathbf{p}_{i}\right\}$, and for each $j<\mathbf{p}_{i}$,

(+) $\quad \mathrm{cl} \operatorname{cz}\left(f_{i j}\right) \subseteq \operatorname{int} Z\left(\mathbf{1}-g_{i j}\right)$ and $\mathrm{cl} \mathrm{cz}\left(g_{i j}\right) \subseteq X \backslash \mathrm{cl} Y_{3-i}$.

By $(+)$, for $j, k<\mathbf{m}, \operatorname{cl} Y_{3-i} \subseteq X \backslash \mathrm{cl} \mathrm{cz}\left(g_{i k}\right)=\operatorname{int} Z\left(g_{i k}\right)$, so

$$
(++) \quad \operatorname{cl} Y_{3-i} \subseteq \cap\left\{\text { int } Z\left(g_{i k}\right): k<j\right\}
$$

(topological operations are with respect to $\tau$ ).

Since $Y_{i}$ is $\left(\mathbf{m}^{+}, \infty, \sigma\right)$-compact, there is a subfamily $\mathcal{F}_{i}$ of $\mathcal{G}_{i}$ with cardinality no greater than $\mathbf{m}$ (which we reindex so that it is $\left\{\left(f_{i j}, g_{i j}: j<\right.\right.$ $\mathbf{m})\}$ such that $V_{i}=\left(\cup\left\{\operatorname{cz}\left(f_{i j}\right): j<\mathbf{m}\right\}\right) \cap Y_{i}$ is $\sigma$-dense in $Y_{i}$. Observe that

$$
W_{i}=\left(\cup\left\{\operatorname{cl}_{\sigma} \operatorname{cz}\left(f_{i j}\right): j<\mathbf{m}\right\}\right) \cap Y_{i}
$$

is a union of fewer than $\mathbf{m}^{+} \sigma$-regular closed subsets of the $\left(\mathbf{m}^{+}, \infty, \sigma\right)$ compact space $Y_{i}$ and is thus $\left(\mathbf{m}^{+}, \infty, \sigma\right)$-compact by 2.12 (a) and (b); further, it contains $V_{i}$, so $W_{i}$ is $\sigma$-dense in $Y_{i}$. Since $\mathcal{H}$ is $(\mathbf{m}, \infty)$-nested, we may assume by 2.4 that $t_{i j}$ is a difjoin of $\left\{g_{i k}: k<j\right\}$, so by 2.9 int $Z\left(g_{i k}\right)=$ $\operatorname{int}\left[\cap\left\{Z\left(g_{i k}\right): k<j\right\}\right]$. Now int $Z\left(t_{i j}\right)$ is $\rho$-dense in $\cap\left\{\right.$ int $\left.Z\left(g_{i k}\right): k<j\right\}$ by this last equality and the $(\mathbf{m}, \rho)$-additivity of $X$. Hence, using $(++)$, we have

$$
Y_{3-i} \subseteq \operatorname{cl}_{\rho} \text { int } Z\left(t_{i j}\right) \text { for each } j<\mathbf{m} .
$$

If $P_{i}=\cup\left\{\right.$ int $\left.Z\left(\left(\mathbf{1}-\left(g_{i j}-t_{3-i, j}\right)\right) \vee 0: j<\mathbf{m}\right)\right\}$, then $P_{1} \cap P_{2}=\phi$ by 2.10 (there setting, for $\mathbf{n}, \mathbf{p}<\mathbf{m}, C=\left\{g_{i j}: j<\mathbf{n}\right\}, s=t_{j, n} D=\left\{g_{2 j}: j<\right.$ $\left.\mathbf{p}\}, t=t_{2, p}\right)$. Now for $i=1,2$, we have by $(+)$ and $(*)$ :

$W_{i} \subseteq\left(\cup\left\{\right.\right.$ int $\left.\left.Z\left(\mathbf{1}-g_{i j}\right): j<\mathbf{m}\right\}\right) \cap Y_{i} \subseteq\left(\cup\left\{\right.\right.$ int $Z\left(\mathbf{1}-g_{i j}\right) \cap$ $\operatorname{cl}_{\rho}$ int $\left.\left.Z\left(\bar{t}_{3-i, j}\right): j<\mathbf{m}\right\}\right)$, which we denote by $U_{i}$. But it can be shown routinely that

$$
\begin{aligned}
& U_{i} \subseteq \cup\left\{\operatorname{cl}_{\rho}\left(\operatorname{int} Z\left(\mathbf{1}-g_{i j}\right) \cap \operatorname{int} Z\left(t_{3-i, j}\right)\right): j<\mathbf{m}\right\}= \\
& \cup\left\{\operatorname{cl}_{\rho} \operatorname{int}\left(Z\left(\mathbf{1}-g_{i j}\right) \cap Z\left(t_{3-i, j}\right)\right): j<\mathbf{m}\right\}= \\
& \cup\left\{\operatorname{cl}_{\rho} \text { int } Z\left(\mathbf{1}-\left(g_{i j}-t_{3-i, j}\right) \vee \mathbf{0}\right): j<\mathbf{m}\right\} \\
& \subseteq \operatorname{cl}_{\rho} P_{i}, \text { completing the proof } . \square
\end{aligned}
$$


2.15. Theorem. Let $\mathcal{H}$ be a completely regular subfamily of $L^{X}$ that is $(\mathbf{m}, \infty)$-nested and $\left(\mathbf{m}^{+}, \mathbf{m}^{+}\right)$-nested for some infinite cardinal $\mathbf{m}$, and suppose $\tau(\mathcal{H})$ is $\mathbf{m}$-additive. Then $\mathbf{m}^{+}$-feebly compact subspace of $X$ are $C^{*}$-embedded in $X$.

Proof. To show that the $\mathbf{m}^{+}$-feebly compact subset $S$ of $X$ is $C^{*}$-embedded, it suffices by 1.17 of [9] to show that completely separated subspaces $Y$ and $Z$ of $S$ are completely separated in $X$. Suppose $f \in C^{*}(S), f[Y]=\{0\}$, and $f[Z]=\{1\}$. Then

$$
Y \subset f^{\leftarrow}\left(-\infty, \frac{1}{3}\right)=U \text { and } Z \subset f^{\leftarrow}\left(\frac{2}{3}, \infty\right)=V .
$$

Observe that $U$ and $V$ are cozero-sets of the $\mathbf{m}^{+}$-feebly compact space $S$, and hence by 2.12 are themselves $\mathbf{m}^{+}$-feebly compact. Clearly they are separated in $X$, and hence by 2.14 are completely $\mathcal{H}$-separated. As $\mathcal{H}$ is completely regular, it follows that $U$ and $V$ are completely separated in $X$. As $Y \subset U$ and $Z \subset V, Y$ and $Z$ are completely separated in $X$, and the theorem is proved.

2.16. Applications. (a) Suppose that $\mathbf{m}=\omega$. Then if $\mathcal{H}$ is a completely regular subfamily of $L^{X}, \tau(\mathcal{H})$ is automatically $\omega$-additive, and $\mathcal{H}$ will be $(\omega, \infty)$-nested if $\mathcal{H}$ is an upper semilattice. As noted above, the $\omega^{+}$feebly compact spaces are usually called weakly Lindelöf spaces, and $\mathcal{H}$ is $\left(\omega^{+}, \omega^{+}\right)$-nested if whenever $\left\{f_{n}\right\}_{n \in \omega}$ and $\left\{g_{n}\right\}_{n \in \omega}$ are sequences of elements of $\mathcal{H}$ such that $f_{k} \leq \mathbf{1}-g_{n}$ for all $n, k<\omega$, then there is an $h \in \mathcal{H}$ such that $f_{k} \leq h \leq \mathbf{1}-g_{n}$ for all $n, k<\omega$. Hence if $\mathbf{m}=\omega, 2.15$ yields that if $\mathcal{H}$ is a completely regular subfamily of $L^{X}$ that is an upper semilattice (i.e. is closed under finite joins), and if $\mathcal{H}$ is $\left(\mathbf{m}^{+}, \mathbf{m}^{+}\right)$-nested, then weakly Lindelöf subspaces of $X$ are $C^{*}$-embedded. In case $\mathcal{H}=C(X,[0,1])$, then by $2.5(\mathrm{~d}) \mathcal{H}$ is $\left(\omega^{+}, \omega^{+}\right)$-nested if and only if $X$ is an $F$-space, and 2.15 says that weakly Lindelöf subspaces of $F$-spaces are $C^{*}$-embedded. This result was known, of course; it appears in [1]. As we will see in the next section, the above result also applies to subspaces of $F$-spaces; this latter may also be inferred indirectly from [1].

(b) Suppose $\mathcal{H} \subset C(X,\{0,1\})$ is basic. As noted in $2.4, \mathcal{H}$ is $(\mathbf{m}, \infty)$ nested if and only if $X$ is $\mathbf{m}$-basically disconnected, and $\tau(\mathcal{H})$ is $\mathbf{m}$-additive if and only if $X$ is a $P_{\mathbf{m}}$-space. Since every union of less than $\mathbf{m}$ clopen 
sets in a $P_{\mathbf{m}}$-space is clopen, every $P_{\mathbf{m}}$-space is $\mathbf{m}$-basically disconnected; as $\beta \omega$ is $\mathbf{m}$-basically disconnected for all $\mathbf{m}$ and has no non-isolated $P$ points (see $12 \mathrm{H}$ of [9]), the converse is false. Thus 2.15 guarantees that if $\{0,1\}^{X}$ contains an $\left(\mathbf{m}^{+}, \mathbf{m}^{+}\right)$-nested completely regular family and if $X$ is a $P_{\mathbf{m}}$-space, then $\mathbf{m}^{+}$-feebly compact subsets of $X$ are $C^{*}$-embedded in $X$. Once $\mathbf{m}>\omega$, insisting that $X$ be a $P_{\mathbf{m}}$-space is a severe restriction that prevents us from applying 2.15 to the space $\beta \omega$ (which is extremally disconnected). Without some restriction, there is no hope of showing that $\mathbf{m}^{+}$-feebly compact subspaces of $\mathbf{m}^{+}$-basically disconnected spaces are $C^{*}$ embedded, since every subspace of $\beta \omega$ is $\mathbf{m}$-feebly compact for sufficiently large $\mathbf{m}$, and $\beta \omega$ is $\mathbf{m}$-basically disconnected for all $\mathbf{m}$; see Section 2 of [26].

(c) In the above, we have considered only complemented families $\mathcal{H} \subseteq$ $L^{X}$. In the next section, we consider noncomplemented families that arise naturally in the study of spaces of minimal prime ideals.

\section{Applications to spaces of minimal prime ideals and subspaces of $F$-spaces.}

In this part of the paper, we apply the theory developed above to give simpler proofs of known results and to derive new results about the class of spaces described in the title of this section. Throughout this section we assume that all hypothesized families of functions $\mathcal{H}$ yield a topology $\tau(\mathcal{H})$ that is Tychonoff (i.e. completely regular and $T_{1}$ ). Note that in $2.2(\mathrm{~d})$ we show that the complete regularity of $\tau(\mathcal{H})$ is equivalent to the (functiontheoretically defined) complete regularity of $\mathcal{H}$. Clearly $\tau(\mathcal{H})$ is $T_{1}$ if and only if for any orderd pair $(x, y)$ of distinct points of $X$, there exists $f_{x, y} \in \mathcal{H}$ such that $f_{x, y}(x)>0$ and $f_{x, y}(y)=0$.

3.1. Definition. Suppose $\mathcal{H} \subseteq L^{X}$ is a completely regular subcollection for some topological space $X$ with topology $\tau(\mathcal{H})$.

(a) If $L=\{0,1\}$ and $\mathcal{H}$ is $\left(\omega^{+}, \infty\right)$-nested, then $\mathcal{B}(\mathcal{H})=\{\mathrm{cz}(f): f \in \mathcal{H}\}$ is called a pretty base for $X$.

(b) If $L=[0,1]$ and $\mathcal{H}$ is $\left(\omega^{+}, \omega^{+}\right)$-nested, then $\mathcal{B}(\mathcal{H})=\{\operatorname{cz}(f): f \in \mathcal{H}\}$ is called a Seever base for $X$.

As noted in $2.5(\mathrm{~d})$, if $X$ is an $F$-space and $\mathcal{H}=C(X,[0,1])$, then $\mathcal{B}(\mathcal{H})$ is a complemented Seever base.

Clearly, if $X$ has a pretty base $\mathcal{B}(\mathcal{H})$, where $\mathcal{H}$ is an $\left(\omega^{+}, \infty\right)$-nested subset of $\{0,1\}^{X}$, then the elements of $\mathcal{B}(\mathcal{H})$ are cozero sets of two-valued 
elements of $C(X,[0,1])$, so every pretty base may be regarded as a Seever base.

Next, we give some background on spaces of minimal prime ideals. See [11], [13], or [12] for more details.

3.2. Definitions and remarks. A commutative ring $R$ whose only nilpotent element is 0 is called reduced. If $S \subseteq R$, let $A(S)=\{a \in R: a S=\{0\}\}$, and let $A(s)=A(\{s\})$ if $\{s\}$ is a singleton. If $R$ is reduced, let $m R$ denote the space of minimal prime ideals of $R$. If $S \subseteq R$, let $h(S)=\{P \in$ $m R: P \supseteq S\}$, let $h^{c}(S)=m R \backslash h(S)$, and abbreviate $h(\{s\})$ by $h(s)$ and $h^{c}(\{s\})$ by $h^{c}(s)$ when $\{s\}$ is a singleton. As is noted in [11], for any $\alpha \in R, h^{c}(\alpha)=h(A(\alpha))$. The hull-kernel topology on $m R$, i.e., the topology whose base is $\left\{h^{c}(\alpha): \alpha \in R\right\}$, is a zero-dimensional Hausdorff topology. We will assume throughout that $m R$ has the hull-kernel topology.

A ring $R$ is said to satisfy the countable annihilator condition (abbreviated CAC) if the family $\{A(a): a \in R\}$ is closed under countable intersection. It is shown in [11] that the (reduced) ring $C(X)$ of continuous real-valued functions on a space $X$ satisfies CAC.

It follows from Lemma 4.2 of [11] that if $R$ is reduced and satisfies CAC, then $\left\{h^{c}(a): a \in R\right\}$ is a pretty base for $m R$. It is shown in Section 6 of [12] that if $R$ is reduced and commutative, then the ring $R[[x]]$ of all formal power series with coefficients in $R$ satisfies CAC. Thus these formal power series rings supply another class of spaces with a pretty base.

Spaces with a pretty base are also discussed in [10], and the theorems in that paper are almost all special cases of the ones given here. The definition of pretty base given here differs superficially from the one given in Definition 2.1 of [10]. The verification of the next proposition is an exercise.

3.3. Proposition. $A$ base $\mathcal{B}$ of a (Tychonoff) space $X$ is pretty if and only if (a) $B \in \mathcal{B}$ implies $B$ is clopen, and $\mathcal{B}$.

(b) if $\left\{B_{n}: n<\omega\right\}$ is a sequence of elements of $\mathcal{B}$, then $\operatorname{cl}\left\{\cup_{n<\omega} B_{n}\right\} \in$

If $Y$ is a subspace of a space $X$, and $\mathcal{H}$ is a completely regular subcollection of $L^{X}$, let $\mathcal{H}_{Y}=\{f \mid Y: f \in \mathcal{H}\}$. We call $\mathcal{B}\left(\mathcal{H}_{Y}\right)$ the trace of $\mathcal{B}(\mathcal{H})$ on $Y$. Note that $\mathcal{B}\left(\mathcal{H}_{Y}\right)$ is a base for the topology of $Y$ since $\mathcal{H}$ is completely regular and hence basic. If $Y$ is dense in $X$, then the map $f \rightarrow f \mid Y$ of 
$\mathcal{H}$ onto $\mathcal{H}_{Y}$ preserves all lattice operators, finitary or infinitary. Hence we have:

3.4. Proposition. If $\mathcal{B}(\mathcal{H})$ is a Seever base or a pretty base on a space $X$, then so is its trace on any dense subspace of $X$.

Indeed, the trace of a Seever base on any subspace of $X$ is a Seever base. As will be seen below, the corresponding assertion for pretty bases is false.

It is easy to verify that the family of all clopen subsets of a basically disconnected space $X$ is pretty base for $X$. In Example 1 of [18], an example is given of a dense subspace $Y$ of a basically disconnected space $X$ that fails to be basically disconnected. By this last proposition, $Y$ has a pretty base. So spaces with a pretty base need not be basically disconnected. Indeed, in [5], an example of minimal prime ideals of a $C(X)$ that fails to be basically disconnected is supplied.

The next proposition enlarges further the class of spaces with a Seever base or a pretty base.

3.5. Proposition. (a) Every subspace of an $F$-space has a Seever base (Cf. 2.5(d)).

(b) Every dense subspace and every open subspace of a space with a pretty base has a pretty base.

Proof. (a) This follows immediately from 3.4 and the fact that $\{\operatorname{cz}(f): f \in$ $C(X,[0,1])\}$ is a Seever base for an $F$-space $X$.

(b) Suppose $Y$ is a subspace of a space $X$ with a pretty base $\mathcal{B}(\mathcal{H})$ for some $\left(\omega^{+}, \infty\right)$-nested completely regular (basic) subfamily of $\{0,1\}^{X}$. If $Y$ is dense, the result follows immediately from 3.4. So let $Y$ be open in $X$. Then $\mathcal{H}_{Y}$ is completely regular. Moreover, if $\left\{B_{n}: n<\omega\right\}$ is a sequence of elements of $\mathcal{B}(\mathcal{H})$, then $\left\{B_{n} \cap Y\right\}$ is a sequence in $\mathcal{B}\left(\mathcal{H}_{Y}\right)$, and since $Y$ is open in $X, \operatorname{cl}_{Y}\left[\cup\left\{B_{n} \cap Y: n<\omega\right\}\right]=Y \cap \operatorname{cl}_{X}\left[\cup\left\{B_{n}: n<\omega\right\}\right]$. Hence $\mathcal{B}\left(\mathcal{H}_{Y}\right)$ is a pretty base for $Y$.

As will be seen in Example 3.7 below, a compact subspace of a space with a pretty base need not have pretty base.

3.6 Proposition. Suppose $X$ is a weakly Lindelöf space. Then

(a) $X$ has a Seever base if and only if $X$ is an $F$-space. 
(b) $X$ has a pretty base if and only if $X$ is basically disconnected.

Proof. (a) The sufficiency is clear by the theorem of Seever cited in 2.5(d). If $X$ is a weakly Lindelöf space, so is any cozero set $C$, and, by 2.15 , a weakly Lindelöf subspace of $X$ is $C^{*}$-embedded. Hence $X$ is an $F$-space.

(b) As was noted above the family of clopen subsets of a basically disconnected space form a pretty base. Conversely, if $X$ has a pretty base $\mathcal{B}$, and $C$ is a cozero set in $X$, then $C$ is weakly Lindelöf, and hence contains a union of countably many members of $\mathcal{B}$ as a dense subspace. Since $\mathcal{B}$ is closed under taking closures of countable unions by Proposition 3.3, the closure of $C$ is open. Thus $X$ is basically disconnected.

3.7. Example. A compact subspace of a space with a pretty base need not have a pretty base. The extremally disconnected space $\beta \omega$ has a pretty base, but by Proposition 3.6(b) its compact subspace $\beta \omega \backslash \omega$ cannot have a pretty base since it fails to be basically disconnected by $6 \mathrm{~W}$ of [9].

A base $\mathcal{B}$ for a topological space $X$ is called proper if $X \notin \mathcal{B}$.

We will see below that spaces that have a proper pretty base are special in character.

3.8. Definitions. (a) If $\mathcal{B}$ is an open base for a space $X$, let $\mathcal{B}^{\prime}$ denote the family of countable unions of elements of $\mathcal{B}$, and if $\alpha X$ is a compactification of $X$, let $K_{\alpha X}(\mathcal{B})=\cap\left\{c \ell_{\alpha X}\left(X \backslash B^{\prime}: B^{\prime} \in \mathcal{B}^{\prime}\right)\right\}$. Then $K_{\alpha X}(\mathcal{B})$ is called the residue of $\mathcal{B}$ in $\alpha X$. (Clearly $K_{\alpha X}(\mathcal{B})$ is a compact subset of $\alpha X \backslash X$, and is nonempty if $\mathcal{B}^{\prime}$ is a proper pretty base.)

(b) If $\mathcal{B}$ is a clopen base for a space $X$, let $\hat{\mathcal{B}}$ denote the Boolean algebra generated by $\hat{\mathcal{B}}$ and let $\operatorname{St}(\hat{\mathcal{B}})$ denote its Stone space (that is, $\operatorname{St}(\hat{\mathcal{B}})$ is the space of ultrafilters on $\hat{\mathcal{B}}$ ). (Note that $\operatorname{St}(\hat{\mathcal{B}})$ is a compactification of $X$ since the map $x \rightarrow\{A \in \hat{\mathcal{B}}: x \in A\}$ embeds $X$ as a dense subspace of $\operatorname{St}(\hat{\mathcal{B}})$ ).

Recall that a nonempty subset $K$ of a Tychonoff space $X$ is called a $P$-set if any $G_{\delta}$ containing $K$ has $K$ in its interior. Equivalently, $K$ is a $P$-set if and only if whenever $K$ is disjoint from a cozero set $V$, we have $K \cap \mathrm{cl}_{X} V=\phi$. For background, see [3].

3.9. Theorem. If $X$ has a pretty base, then the following are equivalent:

(a) $X$ has a proper pretty base,

(b) $X$ fails to be weakly Lindelöf, 
(c) $X$ has a pretty base $\mathcal{B}$ such that if $\alpha X$ is any compactification of $X$ larger than $S t(\hat{\mathcal{B}})$ in the usual ordering of compactifications of $X$ (see 4.2(c) of [23]), then $\alpha X \backslash X$ contains a compact $P$-set of $\alpha X$,

(d) $\beta X \backslash X$ contains a compact $P$-set of $\beta X$.

Proof. (a) implies (b). If $X$ has a pretty base $\mathcal{B}$ and is weakly Lindelöf, choose for each $x \in X$, a $B_{x} \in \mathcal{B}$ such that $x \in B_{x}$; then $\left\{B_{x}: x \in X\right\}$ is an open cover of $X$ which has a countable subfamily $\left\{B_{x(n)}: n<\omega\right\}$ whose union is dense in $X$. Since $X$ is weakly Lindelöf, and $\mathcal{B}$ is pretty, $X=\operatorname{cl}_{X}\left[\cup_{n<\omega} B_{x(n)}\right] \in \mathcal{B}$, so $\mathcal{B}$ is not proper. (b) implies (c). Suppose $\mathcal{B}$ is a proper pretty base for $X$, let $\alpha X$ denote a compactificaton of $X$ larger than $\operatorname{St}(\hat{\mathcal{B}})$, and let $V$ denote a cozero set of $\alpha X$ disjoint from $K=K_{\alpha X}(\mathcal{B})$. Now,

$$
\phi=V \cap K=V \cap\left[\cap\left\{\mathrm{cl}_{\alpha X}\left(X \backslash B^{\prime}\right): B^{\prime} \in \mathcal{B}^{\prime}\right\}\right]
$$

so

$$
\begin{array}{r}
V \subseteq \cup\left\{\alpha X \backslash \operatorname{cl}_{\alpha X}\left(X \backslash B^{\prime}\right): B^{\prime} \in \mathcal{B}^{\prime}\right\} \\
=\cup\left\{\operatorname{int}_{\alpha X}\left\{\alpha X \backslash\left(X \backslash B^{\prime}\right)\right\}: B^{\prime} \in \mathcal{B}^{\prime}\right\}
\end{array}
$$

The locally compact $\sigma$-compact cozero set $V$ is a Lindelöf space, so there is a sequence $\left\{B_{n}^{\prime}: n<\omega\right\}$ in $\mathcal{B}^{\prime}$ such that $V \subset \cup\left\{\right.$ int $_{\alpha X}(\alpha X \backslash(X \backslash$ $\left.B_{n}^{\prime}\right)$ ): $\left.n \in \omega\right\}$. Since each $B_{n}^{\prime}$ is open in $X$, it follows that $X \cap \operatorname{int}_{\alpha X}\{\alpha X \backslash$ $\left.\left(X \backslash B_{n}^{\prime}\right)\right\}=B_{n}^{\prime}$ for each $n<\omega$. So, $V \cap X \subseteq \cup_{n<\omega} B_{n}^{\prime}=B^{\prime}$. Thus

$$
\operatorname{cl}_{\alpha X} V=\operatorname{cl}_{\alpha X}(V \cap X) \subseteq \operatorname{cl}_{\alpha X} B^{\prime}=\operatorname{cl}_{\alpha X}\left(\operatorname{cl}_{X} B^{\prime}\right)=\operatorname{cl}_{\alpha X} B_{0}
$$

where $B_{0}=\operatorname{cl}_{X} B^{\prime} \in \mathcal{B}$ since $\mathcal{B}$ is a pretty base. Also, since $B_{0} \in \mathcal{B} \subseteq$ $\mathcal{B}^{\prime}, K \subseteq \operatorname{cl}_{\alpha X}\left(X \backslash B_{0}\right)$. But both $B_{0}$ and $X \backslash B_{0}$ are in $\hat{\mathcal{B}}$; hence since $\alpha X \geq \operatorname{St}(\hat{\mathcal{B}})$, it follows that $\operatorname{cl}_{\alpha X} B_{0} \cap \operatorname{cl}_{\alpha X}\left(X \backslash B_{0}\right)=\phi$; see 4.2(h) of [23]. Thus $K \cap \operatorname{cl}_{\alpha X} V=\phi$, and we know that $K$ is a $P$-set of $\beta X$. Hence (c) holds. Obviously (c) implies (d).

Suppose (d) holds, $K$ is a compact $P$-set of $\beta X$ contained in $\beta X \backslash X$, and $\mathcal{B}$ is a pretty base for $X$. Let

$$
\mathcal{B}^{*}=\left\{B \in \mathcal{B}:\left(\operatorname{cl}_{\beta X} B\right) \cap K=\phi\right\} .
$$

Since $X \notin \mathcal{B}^{*}$, it suffices to show that $\mathcal{B}^{*}$ is a pretty base for $X$. Suppose $x \in X$ and $V$ is an open neighborhood of $x$. Since $K \subseteq \beta X \backslash X$ is 
closed in $\beta X$, there is an $f \in C(\beta X)$ such that $f(x)=1$ and $f[K]=\{0\}$. Choose $B_{0} \in \mathcal{B}$ such that $x \in B_{0} \subseteq V \cap \operatorname{cz}(f)$. Since $K$ is a $P$-set of $\beta X,\left(\operatorname{cl}_{\beta X} B_{0}\right) \cap K \subseteq \operatorname{cl}_{\beta X}(\operatorname{cz}(f)) \cap K=\phi$. Hence $B_{0} \in \mathcal{B}^{*}$, so $\mathcal{B}^{*}$ is a base for $X$. Suppose $\left\{B_{n}\right\}_{n<\omega}$ is a sequence of elements of $\mathcal{B}^{*}$, in which case $\left(\operatorname{cl}_{\beta X} B_{n}\right) \cap K=\phi$ for each $n<\omega$. Then $S=\cup\left\{\operatorname{cl}_{\beta X} B_{n}: n<\omega\right\}$ is a cozero set of $\beta X$ disjoint from $K$. Since $K$ is a $P$-set, $\left(\operatorname{cl}_{\beta X} S\right) \cap K=\phi$. Thus $\mathcal{B}^{*}$ is a pretty base and (a) holds.

3.10. Corollary. If $X$ has a pretty base, then either $X$ is a weakly Lindelöf basically disconnected space, or $\beta X \backslash X$ contains a compact $P$-set of $\beta X$.

3.11. Remark. In [29], Example 5.1, an $F$-space $X$ is exhibited which is not weakly Lindelöf such that $\beta X \backslash X$ contains no compact $P$-set of $\beta X$. Hence we cannot replace the hypothesis that $X$ have a pretty base in Corollary 3.10 by the weaker assumption that $X$ have a Seever base.

As noted earlier, a space $X$ is called an almost-P-space if each of its zerosets has nonempty interior. Equivalently $X$ is an almost- $P$-space if each of its zerosets is regular closed; see [20]. The next theorem and its proof appear in [10]. We repeat it for the sake of completeness.

3.12. Theorem. A weakly Lindelöf subspace $S$ of an almost $P$-space $X$ with a pretty base is $C$-embedded. If $S$ is also realcompact, then it is closed.

Proof. By Theorem 2.15, $S$ is $C^{*}$-embedded in $X$, so, by 1.18 of [9], we need only show that $S$ is completely separted from any zeroset $Z$ disjoint from it. To see this, find for each $x \in S$, a $B_{x} \in \mathcal{B}$ such that $x \in B_{x} \subseteq X \backslash Z$. Then, since $S$ is weakly Lindelöf, there is a countable subfamily $\left\{B_{x(n)}: n<\omega\right\}$ of the open cover $\left\{B_{x}: x \in S\right\}$ of $S$ such that $B=\operatorname{cl}\left[\cup\left\{B_{x(n)}: n<\omega\right\}\right]$ contains $S$. Now $B \backslash \cup\left\{B_{x(n)}: n<\omega\right\}$ is empty since otherwise it would be a zeroset with empty interior in an almost- $P$-space. Hence $B$ is a clopen subset of $X$ containing $S$ and disjoint from $Z$. Thus $S$ and $Z$ are completely separated. The second assertion follows immediately from 8A.1 of [9].

Recall from [7] that $\beta \omega \backslash \omega$ is an infinite compact $F$-space and an almost- $P$-space. Since it has no countably infinite closed discrete subspace, the conclusion of Theorem 3.12 need not hold for almost- $P$-spaces with a Seever base.

Recall (see 2.1(c)) that if $\mathcal{B}$ is a base for a topological space $(X, \tau)$ then the topology generated by $\left\{X \backslash \mathrm{cl}_{(X, \tau)} B: B \in \mathcal{B}\right\}$ is called the cotopology $\tau^{*}=\tau^{*}(\mathcal{B})$ derived from $\mathcal{B}$. Observe that $\tau^{*} \subseteq \tau$. 
3.13. Definition. If every open cover of $\left(X, \tau^{*}(\mathcal{B})\right)$ has a finite subcover, we call $\mathcal{B}$ a cocompact base.

Suppose $X$ is the space $m R$ of minimal prime ideals of a commutative reduced ring $R$ that satisfies $C A C$. It is shown in Section 5 of [12] that the pretty base $\left(h^{c}(a): a \in R\right)$ is cocompact; that is, the dual hull-kernal topology generated by $\{h(a): a \in R\}$ on $m R$ is quasicompact.

3.14. Theorem. If $(X, \tau)$ has a Seever base $\mathcal{B}(\mathcal{H})$, where $\mathcal{H}$ is a completely regular basic subfamily of $L^{X}$ that is $\left(\omega^{+}, \omega^{+}\right)$-nested, and $\tau^{*}$ is the cotopology derived from $\mathcal{B}(\mathcal{H})$, then, for any weakly Lindelö subspace $Y$ of $X, \tau\left|Y=\tau^{*}\right| Y$.

Proof. As noted above, $\tau^{*}|Y \subseteq \tau| Y$, so it suffices to establish $(+)$ for each $y \in Y$ and $\tau \mid Y$-neighborhood $V$ of $y$, there is an $h \in \mathcal{H}$ such that $y \in$ Int $_{\tau} Z(h) \cap Y \subseteq V$.

To show $(+)$, observe first that since $\mathcal{H}$ is completely regular and basic, $\tau(\mathcal{H})$ and hence $\tau\left(\mathcal{H}_{Y}\right)$ are regular spaces, so we may assume that $V$ is a regular open subset of $Y$. Then $Y \backslash V$ is a regular closed set of $Y$ and hence is weakly Lindelöf by [1].

Since $\mathcal{H}$ is basic, there is an $f \in \mathcal{H}$ such that

$$
y \in Y \cap \operatorname{int} Z(1-f) \subseteq Y \cap \mathrm{cl} \mathrm{cz}(f) \subseteq V
$$

Next choose, for each $z \in Y \backslash \operatorname{clcz}(f)=\operatorname{int} Z(f) \cap Y$, a $g_{z} \in \mathcal{H}$ such that $Z \in \operatorname{int} Z\left(1=g_{z}\right) \subseteq \operatorname{cz}\left(g_{z}\right) \subseteq \operatorname{int} Z(f)$ and note that

(*) $\quad \operatorname{cz}\left(g_{z}\right) \cap \mathrm{cz}(f)=\phi$ for each $z \in Y \backslash \operatorname{cl}(\operatorname{cz}(f))=\operatorname{int} Z(f)$.

Now, $\left\{\operatorname{int} Z\left(\mathbf{1}-g_{z}\right): z \in\right.$ int $\left.Z(f)\right\}$ is an open cover of int $Z(f) \cap Y$ and hence of its weakly Lindelöf subspace $Y \backslash V$. So there is a sequence $\{z(n)\} \subseteq$ $Y \backslash\left(\operatorname{cl}(\operatorname{cz}(f))\right.$ such that if $S=\cup_{n<\omega}$ int $Z\left(\mathbf{1}-g_{z(n)}\right)$, then $S \cap(Y \backslash V)$ is dense in $(Y \backslash V)$. By $\left(^{*}\right)$ and Lemma 2.6, $g_{z(n)} \leq 1-f$ for each $n<\omega$. Thus, since $\mathcal{B}(\mathcal{H})$ is a Seever base, there is an $h \in \mathcal{H}$ such that $g_{z(n)} \leq$ $\mathbf{1}-f$ for each $n<\omega$. Since $\mathcal{H}$ is (completely) regular $S \subseteq Z(\mathbf{1}-h)$ and int $Z(1-f) \subseteq Z(h)$. Thus as $h$ is continuous, it vanishes on a neighborhood of $y$ and is 1 on $Y \backslash V$. Hence $(+)$ holds and the proof of the theorem is complete. 
3.15. Theorem. Suppose $(X, \tau)$ has a cocompact Seever base $\mathcal{B}(\mathcal{H})$, where $\mathcal{H}$ is a completely regular basic subfamily of $C(X,[0,1])$ that is $\left(\omega^{+}, \omega^{+}\right)$-nested, and $Y \subseteq X$ is a weakly Lindelöf subspace of $X$. Then $c_{X} Y$ and $\beta Y$ are homeomorphic. In particular, the closure of any countable subspace of $X$ is compact.

Proof. By Theorem 2.15, $Y$ is $C^{*}$-embedded in $X$, so it suffices to show that $W=\operatorname{cl}_{(X, \tau)} Y$ is compact.

Let $\tau^{*}$ denote the cotopology on $X$ derived from $\mathcal{B}(\mathcal{H})$. We will show next that $W$ is closed in $\left(X, \tau^{*}\right)$.

$(++)$

To see this, choose $p \in X \backslash W$ and a $\tau$-closed neighborhood $U$ of $p$ disjoint from $W$. Since $\mathcal{H}$ is basic, there is an $f \in \mathcal{H}$ such that $p \in$ int $_{\tau} Z(\mathbf{1}-f) \subseteq \mathrm{cz}(f) \subseteq$ int $_{\tau} V$.

For each $x \in W$, pick $g_{X} \in \mathcal{H}$ such that $x \in \operatorname{Int}_{\tau} Z\left(\mathbf{1}-g_{x}\right) \subseteq \operatorname{cz}\left(g_{x}\right) \subseteq$ $X \backslash V$, and note that

$\left(^{*}\right) \mathrm{cz}\left(g_{x}\right) \cap \mathrm{cz}(f)=\phi$ for each $x \in W$.

Now, $\left\{\right.$ int $\left._{\tau} Z\left(1-g_{x}\right) \cap W: x \in W\right\}$ is an open cover of the weakly Lindelöf subspace $W$, so there is a sequence $\{x(n)\}$ such that

$$
D=\cup\left\{\text { int }_{\tau} Z\left(\mathbf{1}-g_{x(n)}\right): n<\omega\right\} \cap W
$$

is dense in $W$. Since $\left(^{*}\right)$ holds, by $2.6 g_{x(n)} \leq 1-f$ for all $n<\omega$. Since $\mathcal{H}$ is $\left(\omega^{+}, \omega^{+}\right)$-nested, there exists $h \in \mathcal{H}$ such that $g_{x(n)} \leq h \leq 1-f$. Then, as in the proof of 3.14 ,

$$
D \subseteq Z(1-h) \text { and } \operatorname{cl}(\operatorname{cz}(f)) \subseteq Z(h) .
$$

Hence $h=1$ on $D$ and hence on $W$, while $h=\mathbf{0}$ on $V$. Consequently $(++)$ holds. Thus, it follows from 3.14 that $W$ is a quasicompact Hausdorff space and hence is compact.

Theorem 3.15 generalizes the corresponding result for spaces with a cocompact pretty base in [10], and thus in turn generalizes the result [5] in case $X=m R$ is the space of minimal prime ideals of a ring without nonzero nilpotents that satisfies CAC. If $X$ is a subspace of an $F$-space, then it has a complemented Seever base $\mathcal{B}$, and the cotopology derived from $\mathcal{B}$ coincides with the original topology, so Theorem 3.13 tells us nothing new in this case. On the other hand, in Section 4 we produce an example of a space with a pretty base (and hence a Seever base) that is not a subspace of an $F$-space. 
Next, we examine locally compact spaces that have a Seever base or a pretty base.

Recall that $X$ is called an $F^{\prime}$-space if disjoint cozero sets of $X$ have disjoint closures. Every $F$-space is an $F^{\prime}$-space, every normal $F^{\prime}$-space is an $F$-space, but there are locally compact $F^{\prime}$-spaces which fail to be $F$-spaces. Also, if every point of $x$ has a neighborhood that is an $F^{\prime}$-space, then $X$ is an $F^{\prime}$-space. For verifications of these assertions, see [3].

The next result is an immediate consequence of Proposition 3.6, the fact that the closure of a weakly Lindelöf subspace is weakly Lindelöf, and the fact that locally basically disconnected spaces are basically disconnected; see [11].

3.16. Theorem. Suppose $X$ is a locally weakly Lindelöf space.

(a) If $X$ has a Seever base, then $X$ is locally an $F$-space, and hence is an $F^{\prime}$-space.

(b) If $X$ has a cocompact pretty base, then $X$ is locally compact and basically disconnected.

Recall from [16] that a locally compact space is said to be substonean if disjoint $\sigma$-compact open subspaces of $X$ have disjoint compact closures. It is established in [16] that $X$ is substonean if and only if $X$ is a compact $F$-space or $X$ fails to be compact and its one-point compactification $\gamma X=X \cup\{\infty\}$ is a compact $F$-space in which $\infty$ is a $P$-point. An example is given in [16] of a substonean space that fails to be an $F$-space. base.

Next, we relate being a substonean space to having a cocompact pretty

3.17. Theorem. If $X$ is a substonean space, then $X$ has a Seever base, and $c l_{X} S$ and $\beta S$ are homeomorphic $F$-spaces for any weakly Lindelöf subspace $S$ of $X$.

Proof. Every substonean space is a subspace of an $F$-space and hence has a Seever base by Proposition 3.4. The weakly Lindelöf subspace $S$ of $X$ is $C^{*}$-embedded in $X$ by Theorem 2.15 . If $X$ is compact, then so is $\operatorname{cl}_{X} S$. Otherwise, let $\gamma X=X \cup\{\infty\}$ denote the one-point compactification of $X$, which, as noted above, is an $F$-space in which $\infty$ is a $P$-point. So each $s \in S$ has a neighborhood $V_{s}$ in $X$ such that $\operatorname{cl}_{X} V_{s}=\operatorname{cl}_{\gamma} X V_{s}$ is compact. The open cover $\left\{V_{s}: s \in S\right\}$ of $S$ has a countable subfamily $\left\{V_{s(n)}: n<\omega\right\}$ 
such that

$$
S \subseteq \operatorname{cl}_{X}\left[\cup\left\{V_{s(n)}: n<\omega\right\}\right] .
$$

Since $U\left\{V_{S(n)}: n<\omega\right\}$ is $\sigma$-compact and hence Lindelöf, its closure in $X$ is compact since $X$ is substonean. Hence $\operatorname{cl}_{X} S$ and $\beta S$ are homeomorphic spaces which are $F$-spaces by Proposition 3.6.

3.18. Theorem. If $X$ is locally weakly Lindelöf and has a cocompact Seever base, then $X$ is substonean.

Proof. By Theorem $3.15, X$ is locally compact. Since $\sigma$-compact open subspaces are weakly Lindelöf, disjoint $\sigma$-compact open subsets are completely separated and hence have disjoint closures. Hence $X$ is substonean.

In [16] a Tychonoff space $X$ is called a Rickart space if each of its $\sigma$-compact open sets has a compact open closure. It is shown in [16] that $X$ is a Rickart space if and only if $X$ is a compact basically disconnected space or $X$ fails to be compact and its one-point compactification $\gamma X=X \cup\{\infty\}$ is basically disconnected and $\infty$ is a $P$-point of $\gamma X$. Moreover, every Rickart space is basically disconnected.

The analogues of Theorems 3.17 and 3.18 for Rickart spaces and spaces with a pretty base follow.

3.19. Theorem. If $X$ is a Rickart space, then $X$ has a pretty base. Moreover, if $S \subseteq X$ is weakly Lindelöf, then $c_{X} S$ and $\beta S$ are homeomorphic.

Proof. The first assertion holds since Rickart spaces are basically disconnected, and the second one follows immediately from Theorem 3.15 and the fact the Rickart spaces are substonean.

3.20. Theorem. If $X$ is locally weakly Lindelöf and has a cocompact pretty base, then $X$ is a Rickart space.

Proof. By Theorems 3.6(b) and 3.15, the closure of any $\sigma$-compact open subspace of $X$ is compact and basically disconnected. So $X$ has a base consisting of compact basically disconnected subspaces and hence is basically disconnected. If $X$ is compact, then it is a Rickart space, so we may assume that $X$ is not compact and write its one-point compactification as $\gamma X=X \cup\{\infty\}$. Since a locally compact locally $F$-space is an $F$-space and has a pretty base, by $3.16 \gamma X$ is a basically disconnected space and by theorem 3.10, $\infty$ is a $P$-point of $\gamma X$. If $S$ is a cozeroset of $\gamma X$ contained in $X$, 
then $\operatorname{cl}_{\gamma X} S=\operatorname{cl}_{X} S$ is compact by Theorem 3.17. If $S$ is a cozero set of $\gamma X$ containing $\infty$, then $X \backslash S=\gamma X \backslash S$ and $S$ are disjoint $\sigma$-compact subsets of the $F$-space $\gamma X$. So, by 2.14 they are completely separated and hence have disjoint closures in $\gamma X$. Since $X \backslash S$ is closed in $\gamma X$, it follows that $\operatorname{cl}_{\gamma X} S$ is open as well as compact. Hence $X$ is a Rickart space.

Recall that a space $X$ is called almost compact if it has a unique compactification. In 6J of [9], it is shown that a Tychonoff space is almost compact if and only if $|\beta X \backslash X| \leq 1$.

3.21. Example. An almost compact $F$-space that is not substonean. Example $E$ of [3] is an almost compact $F^{\prime}$-space that is not a subspace of an $F$-space, and hence cannot be substonean.

3.22. Example. An almost compact extremally disconnected space that is not a Rickart space and hence cannot be the space of minimal prime ideals of a reduced commutative ring satisfying CAC.

Let $X=\beta \omega$ and let $Y=\beta \omega \backslash\{p\}$, where $p$ is any nonisolated point of $\beta \omega$. Then $X$ as well as its dense subspace $Y$ is extremally disconnected, and $p$ is not a $P$-point of $X$ since $\beta \omega \backslash \omega$ is nowhere dense $G_{\delta}$ set of $\beta \omega$ containing $p$. Since $Y$ is almost compact, its one-point compactification is $X$, so $Y$ is not a Rickart space. The last assertion follows from the fact that if a ring $R$ satisfies CAC, then $m R$ has a cocompact pretty base and hence must be a Rickart space by Theorem 3.20.

The final result of this section is related to Theorem 3.17. Its proof is staightforward and hence omitted.

3.23. Theorem. If $X$ is a locally compact noncompact space and $\gamma X=$ $X \cup\{\infty\}$ is its one-point compactification, the following are equivalent.

(a) $\gamma X$ is an $F$-space.

(b) Disjoint open $\sigma$-compact subsets of $X$ have disjoint closures in $X$, at least one of which is compact.

In 2.5(d), we discussed conditions under which a space with a Seever base has an $F$-space compactification. The next section of the paper is devoted to a discussion of a class of examples of spaces with cocompact pretty bases that do not have $F$-space compactifications.

4. Examples of spaces of minimal prime ideals that have no $F$ space compactifications. 
If $X$ is a Tychonoff space, we abbreviate the space $m C(X)$ of minimal prime ideals of $C(X)$ with the hull-kernel topology by $m X$. In Corollary 5.2 of [11] it is shown that $m X$ and $m \beta X\left(=m C^{*}(X)\right)$ are homeomorphic, so whenever it suits us in studying such spaces of minimal prime ideals, we may assume without loss of generality that $X$ is compact. By 2.11 of [9], every prime ideal of $C(X)$ is contained in a unique maximal ideal, and the map $\tau$ of $m X$ onto $\beta X$ that sends each $P \in m X$ to the unique point $p$ of $\beta X$ such that for each $f \in P, p \in \operatorname{cl}_{\beta X} Z(f)$ is continuous. Moreover, $\tau$ maps no proper closed subset of $m X$ onto $\beta X$; if $X$ is an $F$-space, then $\tau$ is a one-one map, and $\tau$ is a homeomorphism if and only if $\beta X$ is basically disconnected. (See Theorem 5.3 of [11] for proofs of the above.) Thus, if $X$ is a compact $F$-space, we may identify the points of $X$ and $m X$ while assigning to $m X$ the topology with the clopen base $\left\{\operatorname{cl}_{X} S: S \in \operatorname{coz} X\right\}$. See Lemma 2 of [5]. Clearly the topology of $m X$ is finer than that of $X$, and $m X$ is a zero-dimensional Hausdorff space (and hence Tychonoff).

We had conjectured at one point that if a space $Y$ had a cocompact pretty base, in particular if $Y=m X$ for some compact space $X$, then some compactification of $Y$ would be an $F$-space. This holds if $Y$ is locally compact by Theorem 3.16 and the fact that the Stone-Cech compactification of a basically disconnected space is basically disconnected. See 5.1 for a related result.

However, there are compact $F$-spaces $X$ for which $m X$ has no compactification that is an $F$-space. The main result in this section (theorem 4.3 ) gives sufficient conditions for a compact $F$-space to be such a space. We then point out, assuming only ZFC, that there are examples of spaces satisfying these conditions; we also show that if Martin's Axiom (MA) is assumed, then $\beta \omega \backslash \omega$ satisfies these conditions.

We begin with some straightforward lemmas. Recall that $P$-sets were defined just before 3.9 .

4.1. Lemma. Let $K$ be a basically disconnected $P$-set of the compact $F$-space $X$. Then $K$ inherits the same topology from $m X$ as it does from $X$.

Proof. It clearly suffices to show that if $C \in \mathrm{cz}(f) X$, then $K \cap \mathrm{cl}_{X} C$ is open in the topology that $K$ inherits from $X$.

We claim that $K \cap \mathrm{cl}_{X} C=\operatorname{cl}_{X}(K \cap C)$. Clearly $\operatorname{cl}_{X}(K \cap C) \subseteq K \cap \mathrm{cl}_{X} C$. Suppose that $p \in K \backslash \operatorname{cl}_{X}(K \cap C)$. Then there exists $V \in \operatorname{coz} X$ such that 
$p \in V$ and $V \cap K \cap C=\phi$. As $V \cap C \in \operatorname{coz} X$ and $K$ is a $P$-set of $X$, it follows that $K \cap \operatorname{cl}_{X}(V \cap C)=\phi$. Thus there exists $W \in \operatorname{coz} X$ such that $p \in W$ and $W \cap V \cap C=\phi$. Thus $p \notin \operatorname{cl}_{X} C$, and hence $K \cap \operatorname{cl}_{X} C \subseteq \operatorname{cl}_{X}(K \cap C)$. Our claim follows.

As $K$ is basically disconnected and $K \cap C \in \operatorname{coz} K$, it follows that $\operatorname{cl}_{K}(K \cap C)$ is open in $K$. (Here $K$ has the topology inherited from $X$ ). But

$$
\mathrm{cl}_{K}(K \cap C)=\operatorname{cl}_{X}(K \cap C)=K \cap \operatorname{cl}_{X} C
$$

by our claim, so $K \cap \mathrm{cl}_{X} C$ is open in the topology $K$ inherits from $X$. The lemma follows.

The following result is likely well-known, but we cannot find a reference for it. Recall that an $F^{\prime}$-space is a Tychonoff space in which disjoint cozero-sets have disjoint closures. Every $F$-space is an $F^{\prime}$-space (see [3]).

4.2. Lemma. If $U$ and $V$ are cozero-sets of the $F^{\prime}$-space $X$, then $c l_{X}(U \cap$ $V)=c l_{X} U \cap c l_{X} V$.

Proof. It suffices to show that $\operatorname{cl}_{X} U \cap \operatorname{cl}_{X} V \subseteq \operatorname{cl}_{X}(U \cap V)$. Let $p \in \operatorname{cl}_{X} U \cap$ $\operatorname{cl}_{X} V$, and let $p \in W \in \operatorname{coz} X$. Then $p \in \operatorname{cl}_{X}(U \cap W)$; for if $T$ is open in $X$ and $p \in T$, then $p \in T \cap W \cap \operatorname{cl}_{X} U$, so $T \cap(U \cap W) \notin \phi$. Thus $\operatorname{cl}_{X}(U \cap W) \cap$ $\operatorname{cl}_{X} V \notin \phi$. As $X$ is an $F^{\prime}$-space, it follows that $U \cap W \cap V \notin \phi$. As $W$ was an arbitrary cozero-set containing $p$, it follows that $p \in \operatorname{cl}_{X}(U \cap V)$.

Recall that almost $P$-spaces were defined just before 3.12. A continuous closed surjection is called irreducible if it maps proper closed subsets of its domain to proper closed subsets of its range. A detailed discussion of irreducible maps can be found in $\S 6.5$ of [23].

4.3. Theorem. Let $X$ be a compact $F$-space that is also an almost $P$ space. Suppose that there exists a closed $P$-set $K$ of $X$ that can be mapped irreducibly onto [0,1]. Then $m X$ is not a subspace of an $F^{\prime}$-space; in particular, $m X$ has no compactification that is an $F$-space.

Proof. As $K$, being compact, is a $C^{*}$-embedded subspace of $X$, there is a continuous surjection $f: X \rightarrow[0,1]$ such that $f \mid K: K \rightarrow[0,1]$ is an irreducible surjection. It follows that $K$ is separable (see $6 \mathrm{~B}(3)$ of [23]). Since $K$ is also an $F$-space (see $6 \mathrm{~L}(4)$ of [23]), it follows that $K$ is extremally disconnected (see $6 \mathrm{~L}(8)$ of [23]) and hence basically disconnected. Hence by $4.1 K$ is a compact subspace of $m X$. 
For each $r \in[0,1]$, we denote $\operatorname{int}_{X} f^{\leftarrow}(r)$ by $A(r)$. Observe that $A(r)$ is clopen in $m X$, and $\operatorname{cl}_{X} A(r)=f^{\leftarrow}(r)$ since $X$ is an almost $P$-space. We claim that $A(r) \cap K=\phi$ for each $r \in[0,1]$. For suppose not; then $K \backslash A(r)$ is a proper closed subset of $K$, and the irreducibility of $f \mid K$ implies that $[0,1] \backslash f[K \backslash A(r)]$ is a nonempty open set of $[0,1]$. It would follow that $\{r\}$ is open in $[0,1]$; from this contradiction we conclude that $A(r) \cap K=\phi$. Let $Y$ be a Tychonoff space containing $m X$ as a subspace. Then $K \operatorname{Cl}_{Y} A(r)=\phi$, since $K$ is a compact subspace of $m X$ and hence of $Y$, and $A(r)$ is a closed subspace of $m X$ disjoint from $K$. Thus there exists $g \in C(Y,[0,1])$ such that $g[A(r)]=\{0\}$ and $g[K]=\{1\}$.

Let $\left(B_{j}\right)_{j \in \omega}$ enumerate an open base for $[0,1]$. We choose by induction on $n$ a sequence $\left(r_{n}\right)_{n \in \omega} \subseteq[0,1]$, a sequence $\left(U_{n}\right)_{n \in \omega}$ of regular open subsets of $X$, and a sequence $\left(g_{n}\right)_{n \in \omega} \subseteq C(Y,[0,1])$ satisfying the following conditions.

(1) $r_{i} \in B_{i / 2}$ or $r_{i} \in B_{i-1 / 2}$, depending on whether $i$ is odd or even.

(2) $f^{\leftarrow}\left(r_{i}\right) \cap K \subseteq U_{i}$.

(3) $g_{i}[K]=\{1\}$ and $g_{i}\left[A\left(r_{i}\right) \cap \operatorname{cl}_{X} U_{i}\right]=\{0\}$.

(4) $i \neq k$ implies $g_{i}^{\leftarrow}\left[\left[0, \frac{1}{4}\right)\right] \cap g_{k}^{\leftarrow}\left[\left[0, \frac{1}{4}\right)\right]=\phi$.

Let $n \in \omega$, and suppose inductively that we have chosen $r_{i}, g_{i}$, and $U_{i}$ for $i<n$ such that (1) to (4) are satisfied if $i, k<n$. Let $j=\frac{n}{2}$ if $n$ is even, and $j=\frac{n-1}{2}$ if $n$ is odd. Choose $p, q \in[0,1]$ such that $p<q$ and $(p, q) \subset B_{j}$. As $f \mid K$ is surjective, there exists $x \in K$ such that $f(x) \in(p, q)$. By induction hypothesis (3),

$$
x \in m X \cap\left[\cap\left\{g_{i}^{\leftarrow}\left(\frac{3}{4}, 1\right]: i<n\right\}\right]
$$

which is open in $m X$. Thus there exists $C \in \operatorname{coz} X$ such that

$$
x \in \operatorname{cl}_{X} C \subset \cap\left\{g_{i}^{\leftarrow}\left(\frac{3}{4}, 1\right]: i<n\right\} .
$$

Then $x \in K \cap \operatorname{cl}_{X} C \cap f^{\leftarrow}(p, q) \subseteq K \cap \operatorname{cl}_{X} C \cap \operatorname{cl}_{X} f^{\leftarrow}[(p, q)]=K \cap \operatorname{cl}_{X}[C \cap$ $\left.f^{\leftarrow}[(p, q)]\right]$; the last equality follows from 4.2. Thus as $K$ is a $P$-set of $X$, it follows that $K \cap C \cap f^{\leftarrow}(p, q) \notin \phi$. As $K$ is extremally disconnected (as noted above) it is zero-dimensional, so there is an open-and-closed subset $B$ of $K$ such that $\phi \neq B \subseteq K \cap C \cap f^{\leftarrow}[(p, q)]$. Thus $B$ is a compact subset of $X$ contained in $C$, so there is a regular open subset $U$ of $X$ such that $B \subseteq U \subseteq \operatorname{cl}_{X} U \subseteq C$. We define $U_{n}$ to be $U$.

As $f \mid K$ is irreducible, there exists $r_{n} \in[0,1] \backslash f[K \backslash B]$. Thus $K \cap f^{\leftarrow}\left(r_{n}\right)=(f \mid K)^{\leftarrow}\left(r_{n}\right) \subseteq B \subseteq U=U_{n}$, and (2) is satisfied when 
$i=n$. Furthermore, since $B \subseteq f^{\leftarrow}[(p, q)]$, it follows that $r_{n} \in(p, q) \subset B_{j}$, so (1) is satisfied when $i=n$.

Since $\operatorname{cl}_{X} U_{n} \subseteq C$, it follows that

$$
\operatorname{cl}_{X} U_{n} \subseteq \cap\left\{g_{i}^{\leftarrow}\left[\frac{3}{4}, 1\right]: i<m\right\} .
$$

It quickly follows that $\operatorname{cl}_{X} U_{n}$ is completely separated in $Y$ from $\cup\left\{g_{i}^{\leftarrow}\left[0, \frac{1}{4}\right]: i<m\right\}$ since $\cap\left\{g_{i}^{\leftarrow}\left[\frac{3}{4}, 1\right]: i<m\right\}$ and $\cup\left\{g_{i}^{\leftarrow}\left[0, \frac{1}{4}\right]: i<m\right\}$ are disjoint zero-sets of $Y$. As noted earlier in the proof, $A\left(r_{n}\right)$ and $K$ are completely separated in $Y$. It follows that $A\left(r_{n}\right) \cap \mathrm{cl}_{X} U_{n}$ is completely separated in $Y$ from

$$
K \cup\left[\cup\left\{g_{i}^{\leftarrow}\left[\left[0, \frac{1}{4}\right]\right]: i<n\right\}\right]
$$

so there exists $g_{n} \in C(Y,[0,1])$ such that $g_{n}\left[A\left(r_{n}\right) \cap \operatorname{cl}_{X} U_{n}\right]=\{0\}$ and

$$
g_{n}\left[K \cup\left[\cup\left\{g_{i}^{\leftarrow}\left[\left[0, \frac{1}{4}\right]\right]: i<m\right\}\right]=\{1\} .\right.
$$

Hence induction assumptions (3) and (4) are satisfied when $i, k \leq n$, and our inductive construction is complete.

To finish the proof it suffices to show that

$$
K \subseteq \operatorname{cl}_{Y} \cup\left[\left\{g_{2_{j}}^{\leftarrow}\left[\left[0, \frac{1}{4}\right)\right]: j \in \omega\right\}\right] \cap \operatorname{cl}_{Y}\left[\cup\left\{g_{2_{j}+1}^{\leftarrow}\left[\left[0, \frac{1}{4}\right)\right]: j \in \omega\right\}\right],
$$

since by (4) above $K$ would be contained in the intersection of $Y$ closures of disjoint cozero-sets of $Y$. We will show that $K \subseteq \operatorname{cl}_{Y}\left[\cup\left\{g_{2_{j}}^{\leftarrow}\left[\left[0, \frac{1}{4}\right)\right]: j \in \omega\right\}\right]$; the other inclusion follows by symmetry.

Let $x \in K$ and let $W$ be a $Y$-neighborhood of $x$. There exists $C \in$ $\operatorname{coz} X$ such that $x \in \operatorname{cl}_{X} C \subset W$. Thus $K \cap \operatorname{cl}_{X} C \neq \phi$ so (as $K$ is a $P$-set) $K \cap C=\phi$, and as above there is a $K$-clopen set $B$ and a regular open

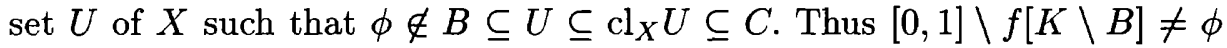
as $f \mid K$ is irreducible, so there exists $j \in \omega$ such $B_{j} \subseteq[0,1] \backslash f[K \backslash B]$. Let $n=2 j$; then $K \cap f^{\leftarrow}\left(r_{n}\right) \subseteq B$, so by (2) $K \cap f^{\leftarrow}\left(r_{n}\right) \subseteq U \cap U_{n}$. Thus $U \cap U_{n} \cap A\left(r_{n}\right) \notin \phi \operatorname{as~cl}_{X} A\left(r_{n}\right)=f^{\leftarrow}\left(r_{n}\right)$. By $(3) g_{n}\left[U \cap \bar{U}_{n} A\left(r_{n}\right)\right]=\{0\}$, so $\phi \neq U \cap g_{2_{j}}^{\leftarrow}\left[\left[0, \frac{1}{4}\right)\right] \subseteq W \cap g_{2_{j}}^{\leftarrow}\left[\left[0, \frac{1}{4}\right)\right]$. Thus $\left[\cup\left\{g_{2_{j}}^{\leftarrow}\left[\left[0, \frac{1}{4}\right)\right]: j \in \omega\right\}\right]$, and our proof is complete.

4.4. Examples. (a) In 1.2 of [19], Kunen shows that if Martin's Axiom is assumed, then the compact almost $P$-space $F$-space $\beta \omega \backslash \omega$ contains a compact subspace $K$ that can be mapped irreducibly onto [0,1]. Hence $i y$ 4.3, Martin's Axiom implies that $m(\beta \omega \backslash \omega)$ cannot be embedded in an 
$F^{\prime}$-space. It is immediate that any space containing $m(\beta \omega \backslash \omega)$ as an openand-closed subspace, such as $m(\beta D \backslash D)$ where $D$ is an infinite discrete space, cannot be embedded in an $F^{\prime}$-space under Martin's Axiom.

(b) Let $X$ be any locally compact $\sigma$-compact noncompact space. By $6 \mathrm{~L}(2)$ or [23], $\beta X \backslash X$ is a compact $F$-space that is an almost $P$-space. As $X$ is not pseudocompact it contains a closed $C$-embedded countably infinite discrete subspace $D$ (see 1.21 of [9]). By 2.1 of [21], $\operatorname{cl}_{\beta X} D \backslash X$ is a $P$-set of $\beta X \backslash X$, and $\operatorname{cl}_{\beta X} D \backslash X \cong \beta \omega \backslash \omega$. If we assume Martin's Axiom, there is a $P$-set $K$ of $\operatorname{cl}_{\beta X} D \backslash X$ that can be mapped irreducibly onto [0,1] (see (a) above), and $K$ is evidently a $P$-set of $\beta X \backslash X$. Hence $m(\beta X \backslash X)$ cannot be embedded in an $F^{\prime}$-space.

(c) It has been announced in [8] that is relatively consistent with ZFC that $\beta \omega \backslash \omega$ has no compact $P$-set $K$ that can be mapped irreducibly onto $[0,1]$. As this is written, it is not known whether it is a theorem in ZFC that $m(\beta \omega \backslash \omega)$ has no $F$-space compactification. In [4] the axiom "Mel" is formulated, and it is shown that "Mel" implies that $m(\beta \omega \backslash \omega)$ is not basically disconnected; it is not known if "Mel" is a theorem of ZFC.

(d) In Corollary 6 of [5], an example is produced in ZFC of a quotient $K$ of $\beta \omega \backslash \omega$ that is a compact $F$-space and almost $P$-space, and which contains a $P$-set that maps irreducibly onto $[0,1]$. Hence by $4.3, m K$ cannot be embedded in an $F^{\prime}$-space. This shows that in ZFC there are spaces with cocompact pretty bases that cannot be embedded in $F^{\prime}$-spaces, and in particular do not have $F$-space compactifications.

\section{Miscellaneous results and open problems.}

It seems natural to ask: when does a space with a cocompact pretty base have a basically disconnected compactification? Theorem 5.1 translates this query into the language of Boolean algebras, and in doing so reinforces the "naturalness" of the concept of a pretty base. Recall that for any space $X, \mathcal{B}(X)$ denotes the Boolean algebra of clopen subsets of $X$.

5.1. Theorem. A space $X$ has a basically disconnected compactification if and only if there is a subalgebra $\mathcal{A}$ of $\mathcal{B}(X)$ that is a pretty base for $X$.

Proof. Suppose $X$ has such a pretty base $\mathcal{A}$, and let $K$ denote the Stone space of $\mathcal{A}$. By identifying $X$ with the fixed ultrafilters on $\mathcal{B}(X)$, we may regard $K$ as a compactification of the zero-dimensional space $X$. Clearly $\mathcal{B}(K)$ is a Boolean algebra isomorphic with $\mathcal{A}$, and clearly $\mathcal{A}$ is countably 
complete since it is a pretty base. Thus $\mathcal{B}(K)$ is also countably complete, whence $K$ is basically disconnected.

Conversely if $X$ has a basically disconnected compactification $K$, then, as noted in the proof of $3.6, \mathcal{B}(K)$ is a pretty base for $K$. The trace of $\mathcal{B}(K)$ on $X$ is a pretty base by $3.5(\mathrm{~b})$, and is clearly a subalgebra of $\mathcal{B}(X)$. So the theorem holds.

We know that a Tychonoff space $X$ is an $F$-space, or basically disconnected, or extremally disconnected if and only if $\beta X$ has the corresponding property. No simple relationship of this sort exists for a space with a pretty base; 5.1 above is the best we can do (note that in $4.4(\mathrm{~d})$, we produced a space that has a cocompact pretty base but which has no $F$-space compactification, and, in particular, no basically disconnected compactification.

Hence we are motivated to ask:

5.2. Problem. Is there a "nice" topological property $\mathcal{P}$ such that a Tychonoff space $X$ has a pretty base if and only if it has a compactification with property $\mathcal{P}$ ?

5.3. Remark. Recall from [14] and [15] that $X$ is called a quasi-F-space if dense cozero sets of $X$ are $C^{*}$-embedded in $X$. If $X$ is any compact space, there is quasi- $F$-space $\mathrm{QF}(\mathrm{X})$ and a continuous irreducible surjection $f$ of $\mathrm{QF}(\mathrm{X})$ onto $X$ such that if $Y$ is any compact quasi- $F$-space that maps onto $Y$ under a continuous irreducible surjection, then $Y$ maps irreducibly onto $Q F(X)$. Thus $Q F(X)$ is called the quasi-F-cover of $X$. In unpublished work, Johannes Vermeer has shown that $m X$ and $m(Q F(X))$ are homeomorphic if $X$ is compact. Hence, in the study of spaces of minimal prime ideals of rings $C(X)$, one may assume without loss of generality that $X$ is a compact quasi- $F$-space.

5.4. Problem. Suppose $X$ is a space with a cocompact pretty base. Is there a ring $\mathcal{R}$ satisfying $C A C$ such that $X$ and $m R$ are homeomorphic? Indeed, if the answer is affirmative, may one always assume that $R=C(Y)$ for some (compact quasi- $F$ ) space $Y$ ?

5.5. Problem. Suppose $X$ has a cocompact pretty base and $X$ is basically disconnected. Does it follow that $X$ is locally compact? In particular, suppose $X=m Y$ for some (compact) space $Y$, and $X$ is basically disconnected. Does it follow then that $X$ is locally compact? 
As we saw in Section 3, the extremally disconnected locally compact space obtained by removing a nonisolated point from $\beta \omega$ cannot have a cocompact pretty base, so even an affirmative answer would not provide a characterization of basically disconnected spaces with a cocompact pretty base.

The minimal basically disconnected cover described in [V] may be a valuable tool in any attempt to solve 5.5 .

There are many other mysteries about spaces with a cocompact pretty base. In particular, we pose the following.

5.6. Problem. Suppose $X$ has a cocompact pretty base. Must $X$ be normal, or a $k$-space, or strongly zero-dimensional?

\section{REFERENCES}

1. Comfort, W., Hindman, N., and Negrepointis, S., $F^{\prime}$-spaces and their product with $P$-spaces, Pacific J. Math 28 (1969), 489-502.

2. Comfort, W. and Negrepointis, S., The Theory of Ultrafilters, Springer-Verlag, New York, 1974.

3. Dow, A., F-spaces and $F^{\prime}$-spaces, Pacific J. Math. 108 (1983), 275-283.

4. - The space of minimal prime ideals of $C(\beta N \backslash N)$ is probably not basically disconnected, General Topology and Applications (Proceedings of the 1988 Northeast Conference), Marcel Dekrer Lecture Notes in Pure and Applied Mathematics Vol. 123 (1990), 81-86.

5. Dow, A., Henriksen, M., Kopperman, R., and Vermeer, J., The space of minimal prime ideals need not be basically disconnected, Proc. Amer. Math. Soc. 104 (1988), 317-320.

6. The countable annihilator condition and weakly Lindelof subspaces of minimal prime ideals, Proc. IMM Conference in Baku, USSR (1987), to appear.

7. Fine, N. and Gillman, L., Extensions of Continuous functions in $\beta N$, Bull. Amer. Math. Soc. 66 (1960), 376-381.

8. Frankiewciz, R., Shelah, S., and Zbierski, B., Embeddings of Boolean Algebras in $P(\omega)$ mod finite, Abstracts Amer. Math. Soc. 10 (1989), 399.

9. Gillman, L. and Jerison, M., Rings of Continuous Functions, D. Van Nostrand Publ. Co., Princeton, N.J. 1960.

10. Henriksen, M., Spaces with a pretty base, J. Pure Appl. Algebra 70 (1991), 81-87.

11. Henriksen, M. and Jerison, M., The space of minimal prime ideals of a commutative ring, Trans. Amer. Math. Soc. 115 (1965), 110-130.

12. Henriksen, M. and Kopperman, R., A general theory of structure theory with applications to spaces of prime ideals, Algebra Universalis, to appear.

13. Huckaba, J., Commutative Rings with Zero Divisors, Marcel Dekker Publ. Co., New York, 1988. 
14. Henriksen, M., Vermeer, J., and Woods, R.G., Quasi-F-covers of Tychonoff Spaces, Trans. Amer. Math. Soc. 303 (1987), 779-803.

15. , Wallman Covers of compact-spaces, Dissertationes Math. 280 (1989), 1-31.

16. Henriksen, M. and Woods, R.G., General Topology as a tool in functional analysis, Annals of the New York Academy of Sciences 552 (1989), 60-69.

17. Juhasz, I., Cardinal Functions in topology, Mathematische Centrum Tracts. No. 34, Amsterdam, 1991.

18. Kohls, C., Hereditary properties of some special spaces, Arch. Math. (Basel) 12 (1961), 129-133.

19. Kunen, K., Some points in $\beta N$, Proc. Camb. Philos. Soc. 80 (1976), 385-398.

20. Levy, R., Almost P-spaces, Canad. J. Math. 29 (1977), 284-288.

21. van Mill, J., Weak P-points in Cech-Stone compactifications, Trans. Amer. Math. Soc. 283 (1982), 657-678.

22. — An introduction to $\beta \omega$, Handbook of Set-Theoretic Topology, North Holland Publ. Co. 1984, 503-567.

23. Porter, J. and Woods, R.G., Extensions and Absolutes of Topological Spaces, SpringerVerlag, New York, 1987.

24. Seever, G.L., Measures on F-spaces, Trans. Amer. Math. Soc. 133 (1968), 267-280.

25. Stephenson, R.M., Initially $k$-compact and related spaces, Handbook of Set-Theoretic Topology, North Holland Publ. Co., 1987, 603-632.

26. Swardson, M., A generalization of $F$-spaces and some topological characterizations of GCH, Trans. Amer. Math. Soc. 279 (1983), 661-675.

27. Vermeer, J., The smallest basically disconnected compactification of a space, Topology and Appl. 17 (1984), 217-232.

28. Willard, S., General Topology, Addison-Wesley Publ. Co., Waltham, MA, 1970.

29. Dow, A., and Förster, O., Absolute $C^{*}$-embedding of F-spaces, Pacific J. Math. 98 (1982), 63-71.

York University, North York, Ontario, Canada

Harvey Mudd College College, Claremont, California, U.S.A.

City College of New York, New York, New York, U.S.A.

The University of Manitoba, Winnipeg, Manitoba, Canada 